\title{
Outline of Hadronic Mathematics, Mechanics and Chemistry as Conceived by R. M. Santilli
}

\author{
Richard Anderson \\ The R. M. Santilli Foundation, Palm Harbor, Florida, U.S.A.
}

Email address:

board@santilli-foundation.org

\section{To cite this article:}

Richard Anderson. Outline of Hadronic Mathematics, Mechanics and Chemistry as Conceived by R. M. Santilli. American Journal of Modern Physics. Special Issue: Issue II: Foundations of Hadronic Mechanics. Vol. 5, No. 2-1, 2016, pp. 1-16.

doi: 10.11648/j.ajmp.2016050201.11

Received: August 11, 2015; Accepted: August 24, 2015; Published: May 18, 2016

\begin{abstract}
In this paper, we outline the various branches of hadronic mathematics and their applications to corresponding branches of hadronic mechanics and chemistry as conceived by the Italian-American scientist Ruggero Maria Santilli. According to said conception, hadronic mathematics comprises the following branches for the treatment of matter in conditions of increasing complexity: 1) 20th century mathematics based on Lie's theory; 2) IsoMathematics based on Santilli's isotopies of Lie's theory; 3) GenoMathematics based on Santilli's formulation of Albert's Lie-admissibility; 4) HyperMathematics based on a multi-valued realization of genomathematics with classical operations; and 5) HyperMathematics based on Vougiouklis $\mathrm{H}_{\mathrm{v}}$ hyperstructures expressed in terms of hyperoperations. Additionally, hadronic mathematics comprises the anti-Hermitean images (called isoduals) of the five preceding mathematics for the description of antimatter also in conditions of increasing complexity. The outline presented in this paper includes the identification of represented physical or chemical systems, the main mathematical structure, and the main dynamical equations per each branch. We also show the axiomatic consistency of various branches of hadronic mathematics as sequential coverings of 20th century mathematics; and indicate a number of open mathematical problems. Novel physical and chemical applications permitted by hadronic mathematics are presented in subsequent collections.
\end{abstract}

Keywords: Santilli isomathematics, Genomathematics, Hypermathematics

\section{20th Century Mathematics, Mechanics and Chemistry}

\subsection{Represented Systems}

Single-valued, closed-isolated, time-reversible systems of point-like particles moving in vacuum solely under action at a distance Hamiltonian interactions, such as the structure of atoms and molecules.

\subsection{Main Mathematical Structure}

Basic unit

$$
I=+1
$$

Basic numeric fields $\mathrm{n}=$ real, complex, quaternionic numbers

$$
F(n, \times, 1), n
$$

Basic Associative product

$$
n m=n \times m, 1 \times n=n \times 1=n \forall n \in F
$$

Measurement units of time, energy, etc. all positive Ordinary functional analysis $f(r) \in F$,

Ordinary differential calculus

Conventional Lie theory

$$
\begin{gathered}
{\left[X_{i}, X_{j}\right]=X_{i} \times X_{j}-X_{j} \times X_{i}==C_{i j}^{k} \times X_{k},} \\
A(w)=e^{X \times w \times i} \times A(0) \times e^{-i \times w \times X} .
\end{gathered}
$$

Euclidean geometry and topology

$$
\begin{gathered}
E(r, \delta, 1), r=\left(r^{k}\right), k=1,2,3, \delta=\operatorname{Diag} \cdot(1,1,1), \\
r^{2}=r^{i} \times \delta_{i j} \times r^{j}=r_{1}^{2}+r_{2}^{2}+r_{3}^{2} \in F,
\end{gathered}
$$


Minkowskian geometry

$$
\begin{gathered}
M(x, \eta, I): x=\left(x^{\mu}\right), \mu=1,2,3,4, x^{4}=t, \\
\eta=\text { Diag. }\left(+1,+1,+1,-c^{2}\right), \\
x^{2}=x^{\mu} \times \eta_{\mu \nu} \times x^{v}=x_{1}^{2}+x_{2}^{2}+x_{3}^{2}-t^{2} c^{2} \in F,
\end{gathered}
$$

Riemannian geometry

$$
\begin{gathered}
R(x, g(x), I): x=\left(x^{\mu}\right), \mu=1,2,3,4, x^{4}=t, \\
x^{2}=x^{\mu} \times g(x)_{\mu \nu} \times x^{\nu} \in F \\
x^{2}=x^{\mu} \times g(x)_{\mu \nu} \times x^{\nu} \in F
\end{gathered}
$$

Symplectic geometry.

$$
\omega=d r^{k} \wedge d p_{k}
$$

\subsection{Dynamical Equations}

Newton equation

$$
m \times \frac{d v}{d t}-F^{S A}(t, r, v,)=0,
$$

Variational principle

$$
\delta A=\delta \int\left(p_{k} \times d r^{k}-H \times d t\right)=0 .
$$

Hamilton's equations without external terms

$$
\frac{d r^{k}}{d t}=\frac{\partial H(r, p)}{\partial p_{k}}, \quad \frac{d p_{k}}{d t}=-\frac{\partial H(r, p)}{\partial r^{k}}
$$

Hilbert space $H$ over $C$ with states $|\psi\rangle$ over $(C)$

Expectation value of a Hermitean operator $A$

$$
<A>=<\psi|\times A \times| \psi>\in C,
$$

Heisenberg equation

$$
i \times \frac{d A}{d t}=[A, H]=A \times H-H \times A,
$$

Schrödinger equations

$$
\begin{aligned}
& H \times|\psi>=E \times| \psi> \\
& p \times\left|\psi>=-i \times \partial_{r}\right| \psi>
\end{aligned}
$$

Dirac equation

$$
\begin{aligned}
& \left(\eta^{\mu \nu} \times \gamma_{\mu} \times p_{v}-i \times m \times c\right) \times \mid \psi>=0 \\
& \left\{\gamma_{\mu}, \gamma_{v}\right\}=\gamma_{\mu} \times \gamma_{\nu}+\gamma_{n} u \times \gamma_{\mu}=2 \times \eta_{\mu \nu}
\end{aligned}
$$

Comments and References

The literature on 20th century mathematics, mechanics and chemistry is so vast and so easily identifiable to discourage discriminatory partial listings.

\section{Isomathemates, Isomechanics and Isochemistry}

\subsection{Represented Systems [1-5]}

Single-value, closed-isolated, time-reversible system of extended-deformable particles with action at a distance Hamiltonian and contact non-Hamiltonian interactions, such as the structure of hadrons, nuclei and stars, in the valence electron bonds and other systems.

\subsection{Main Mathematical Structure s [1-5]}

Santilli IsoUnit $\hat{I}$ and isotopic element $\widehat{T}^{1}$

$$
\hat{I}=\hat{I}(r, p, a, \psi, \ldots)=1 / \hat{T}(r, p, a, \psi, \ldots)>0,
$$

Santilli IsoFields

$$
\hat{F}(\hat{n}, \widehat{x}, \hat{I}), \hat{n}=n \times \hat{I},,
$$

Santilli isoproduct

$$
\begin{gathered}
\hat{n} \widehat{\times} \hat{m}=\hat{n} \times \hat{T} \times \hat{m} \in \hat{F}, \\
\hat{I} \hat{\times} \hat{n}=\hat{n} \widehat{\times} \hat{I}=\hat{n} \forall \hat{n} \in \hat{F},
\end{gathered}
$$

Representation via the isotopic element of extended-deformable particles under non-Hamiltonian interactions

$$
\widehat{T}=\operatorname{Diag} \cdot\left(\frac{1}{n_{1}^{2}}, \frac{1}{n_{2}^{2}}, \frac{1}{n_{3}^{2}}\right) \times e^{\Gamma(r, p, \psi, \partial \psi, \ldots)}
$$

IsoCoordinates $\hat{r}=r \times \hat{I} \in \hat{F}$,

IsoFunctional analysis $\hat{f}(\hat{r})=f(\hat{r}) \times \hat{I} \in \hat{F}$,

IsoDifferential Calculus

$$
\begin{array}{r}
\hat{d} \hat{r}=d r+r \times \hat{T} \times d \hat{I}, \\
\frac{\hat{\partial} \hat{f}(\hat{r})}{\partial \hat{r}}=\hat{I} \times \frac{\partial \hat{f}(\hat{r})}{\partial \hat{r}},
\end{array}
$$

Santilli Lie-Isotopic Theory

$$
\begin{gathered}
{\left[X_{i}, X_{j}\right]=X_{i} \widehat{\times} X_{j}-X_{j} \widehat{\times} X_{i}==C_{i j}^{k}(r, p, \ldots) \times X_{k},} \\
A(w)=\hat{e}^{X \times w \times i} \widehat{\times} A(0) \hat{\times} \hat{e}^{-i \times w \times X} .
\end{gathered}
$$

Santilli Iso-Euclidean Geometry

$$
\begin{gathered}
\hat{E}(\hat{r}, \hat{\delta}, \hat{I}), \hat{\delta}(r, p, z, \psi, \ldots)=\hat{T}(r, p, z, \psi, \ldots) \times \delta, \\
\hat{T}=\operatorname{Diag} \cdot\left(1 / n_{1}^{2}, 1 / n_{2}^{2}, 1 / n_{3}^{2}\right), \\
\hat{r}^{\widehat{2}}=\hat{r}^{i} \hat{\times} \hat{\delta}_{i j} \widehat{\times} \hat{r}^{j}=\left(\frac{r_{1}^{2}}{n_{1}^{2}}+\frac{r_{2}^{2}}{n_{2}^{2}}+\frac{r_{3}^{2}}{n_{3}^{2}}\right) \times \hat{I} \in \hat{F},
\end{gathered}
$$

${ }^{1}$ See Santilli's curriculum

http://www.world-lecture-series.org/santilli-cv Prizes and Nominations

http://www.santilli-foundation.org/santilli-nobel-nominations.html and scientific archive

http://www.santilli-foundation.org/news.html 
Santilli Iso-Minkowskian Geometry

$$
\begin{array}{r}
\hat{M}(\hat{x}, \hat{\eta}, \hat{I}): \hat{x}=\left(\hat{x}^{\mu}\right), \mu=1,2,3,4, x_{4}=t, \\
\hat{\eta}(x, \psi, \ldots)=\hat{T}(x, \psi, \ldots) \times \eta, \\
\hat{T}=\operatorname{Diag} \cdot\left(1 / n_{1}^{2}, 1 / n_{2}^{2}, 1 / n_{3}^{2}, 1 / n_{4}^{2}\right), \\
\hat{x}^{\widehat{2}}=\hat{x}^{\mu} \widehat{\times} \hat{\eta}_{\mu \nu} \widehat{\times} \hat{x}^{v}=\left(\frac{x_{1}^{2}}{n_{1}^{2}}+\frac{x_{2}^{2}}{n_{2}^{2}}+\frac{x_{3}^{2}}{n_{3}^{2}}-t^{2} \frac{c^{2}}{n_{4}^{2}}\right) \times \hat{I} \in \hat{F},
\end{array}
$$

Santilli Iso-Riemannian Geometry

$$
\begin{aligned}
& \hat{R}(\hat{x}, \hat{g}, \hat{I}): \hat{g}=\hat{T}(x, v, \ldots) \times g(x), \\
& \hat{x}^{\widehat{2}}=\left(\frac{g_{11}}{n_{1}^{2}}+\frac{g_{22}}{n_{2}^{2}}+\frac{g_{33}}{n_{3}^{2}}-\frac{g_{44}}{n_{4}^{2}}\right) \times \hat{I} \in \hat{F},
\end{aligned}
$$

Santilli Iso-Symplectic Geometry

$$
\widehat{\omega}=\hat{d} \hat{r}^{k} \widehat{\Lambda} \hat{d} \hat{p}_{k}
$$

\subsection{IsoDynamical IsoEquations s [1-5]}

Newton-Santilli IsoEquation

$$
\begin{array}{r}
\hat{m} \widehat{\times} \frac{\hat{d} \hat{v}}{\hat{d} \hat{t}}-F^{S A}(t, r, p)= \\
m \times \frac{d v}{d t}-F^{S A}(t, r, p)-F^{N S A}(t, r, p, \ldots)=0,(42)
\end{array}
$$

IsoVariational principle

$$
\hat{\delta} \hat{A}=\hat{\delta} \widehat{\int}\left(\hat{p}_{k} \widehat{\times} \hat{d} \hat{r}^{k}-\widehat{H} \widehat{\times} \hat{d} \hat{t}\right)=0 .
$$

Hamilton-Santilli IsoEquations

$$
\frac{\widehat{d} \hat{r}^{k}}{\hat{d} \hat{t}}=\frac{\widehat{\partial} \widehat{H}(\hat{r}, \hat{p})}{\widehat{\partial} \hat{p}_{k}}, \quad \frac{\widehat{d} \hat{p}_{k}}{\hat{d} \hat{t}}=-\frac{\widehat{\partial} \widehat{H}(\hat{r}, \hat{p})}{\widehat{\partial} \hat{r}^{k}}
$$

Iso-Hilbert space $\widehat{H}$ over $C$ with states $|\widehat{\psi}\rangle$ over the isofield $\hat{C}^{2}$

IsoExpectation value of a Hermitean operator $\hat{A}$ on $\widehat{H}$

$$
<\hat{A}>=<\hat{\psi}|\hat{\times} \hat{A} \widehat{\times}| \hat{\psi}>\in \hat{C}
$$

Heisenberg-Santilli IsoEquation

${ }^{2}$ As shown in the seminal paper [6] of 1982 , but vastly ignored for the past four decades, isomechanics formulated on iso-Hilbert spaces over isofields eliminates the divergencies of quantum mechanics and related scattering theories. This important feature is primarily due to the fact that, for all physical and chemical applications worked out to date, the isounit $\hat{I}=1 / \hat{T}>0$ must have a large value of the exponential type (27) and, consequently, the isotopic element $\hat{T}$ must have a very small value. This occurrence eliminates the singularity of the Dirac delta "distribution" when lifted to the Dirac-Myung-Santilli delta "isofunction" as shown by the realization of the type

$$
\hat{\delta}\left(r-r_{0}\right)=\frac{1}{2 \pi} \int_{-\infty}^{+\infty} e^{i k \hat{T}\left(r-r_{o}\right)} d k,
$$

with $\hat{T}=\frac{N}{r-r_{0}}, N \ll 1$. Similarly, perturbative and other series with Hermitean operators that are divergent or slowly convergent in quantum mechanics can be lifted into isoseries of the type

$$
A(w)=\hat{I}+\frac{w(A \widehat{T} H-H \widehat{T} A)}{1 !}+\cdots
$$

that are manifestly convergent for $w>1$ but $\hat{T} \ll w$.As shown by A. O. E. Animalu and R. M. Santilli in five papers published proceedings [25], the above lack of divergences carries over to the covering of the scattering theory known as isoscattering theory, by therefore achieving numerical results without the use of infinities for the renormalization of divergent series.

$$
\begin{array}{r}
\hat{\imath} \widehat{\times} \frac{\hat{d} \hat{A}}{\hat{d} \hat{t}=}[\hat{A}, \widehat{H}]=\hat{A} \widehat{\times} \widehat{H}-\widehat{H} \widehat{\times} \hat{A}=\hat{A} \times \hat{T}(\hat{\psi}, \ldots) \times \\
\hat{H}(\hat{r}, \hat{p})-\widehat{H}(\hat{r}, \hat{p}) \times \hat{T}(\hat{\psi}, \ldots) \times \hat{A}
\end{array}
$$

Schrödinger-Santilli IsoEquation

$$
\begin{gathered}
\widehat{H} \widehat{\times}|\hat{\psi}>=\hat{H}(\hat{r}, \hat{p}) \times \hat{T}(\hat{\psi}, \hat{\partial} \hat{\psi}, \ldots) \times| \hat{\psi}>=\hat{E} \widehat{\times} \mid \hat{\psi}>= \\
E \times \mid \hat{\psi}>, \\
\hat{p} \widehat{\times}\left|\hat{\psi}>=-\hat{\imath} \widehat{\times} \hat{\partial}_{\hat{r}}\right| \hat{\psi}>=-i \times \hat{I} \times \partial_{\hat{r}} \mid \hat{\psi}>,
\end{gathered}
$$

Dirac-Santilli IsoEquation

$$
\begin{gathered}
\left(\hat{\eta}^{\mu \nu} \widehat{\times} \hat{\gamma}_{\mu} \widehat{\times} \hat{p}_{v}-\hat{\imath} \widehat{\times} \hat{m} \widehat{\times} \hat{c}\right) \widehat{\times} \mid \hat{p} s i>=0 . \\
\left\{\hat{\gamma}_{\mu}, \hat{\gamma}_{\nu}\right\}=\hat{\gamma}_{\mu} \widehat{\times} \hat{\gamma}_{\nu}+\hat{\gamma}_{\nu} \widehat{\times} \hat{\gamma}_{\mu}=\hat{2} \widehat{\times} \hat{\eta}_{\mu \nu}=2 \times \hat{\eta}_{\mu \nu},
\end{gathered}
$$

\subsection{Comments and References}

As it is well known, the local-differential calculus of 20th century mathematics can solely represent a finite set of isolated dimensionless points. In view of this structural feature, Newton formulated his celebrated equations (14) for massive points, resulted in a conception of nature that was adopted by Galileo and Einstein, became the dominant notion of 20th century sciences, and was proved to be valid for classical or quantum particles moving in vacuum at large mutual distances, such as for our planetary system or the atomic structure.

However, when bodies move within physical media, such as for a spaceship during re-entry in our atmosphere or for a proton in the core of a star, point-like abstractions of particles became excessive, e.g., because a macroscopic collection of point-particles cannot have entropy (since all known Hamiltonian interactions are invariant under time reversal), with consequential violation of thermodynamical laws and other insufficiencies.

Besides the clear identification of these insufficiencies, the first historical contribution by the Italian-American scientist Ruggero Maria Santilli (see Footnote 1) has been the generalization of 20th century mathematics into such a form to admit a time invariant representation of extended, and therefore deformable particles under conventional Hamiltonian as well as contact non-Hamiltonian interactions, with implications for all quantitative sciences.

The above central objective was achieved in monographs [1] originally written by Santilli during his stay at MIT from 1974 to 1977 (where they appeared as MIT preprints). Monographs [1] were then completed by Santilli during his stay at Harvard Universityfrom 1977 to 1982 under DOE support, and released for publication only following the delivery at Harvard of a post Ph. D. seminar Course in the field.

The representation of extended-deformable bodies moving within physical media was achieved via an axiom-preserving lifting, called isotopy, of the conventional associative product $A B=A \times B$ between generic quantities $A, B$ (such as numbers, functions, matrices, operators, etc.) into the form $A \widehat{\times} B=A \times \widehat{T} \times B$, Eq. (25). Conventional interactions are represented via conventional Hamiltonian, while actual shape and non-Hamiltonian interactions are represented via realization of the quantity $\widehat{T}$, called isotopic element, of the 
type (27).

Santilli then achieved in monographs [1] the axiom-preserving isotopies of the various branches of Lie's theory, e.g., Eqs. (30), (31,) including their elaboration via the initiation of the isotopies of functional analysis. In particular, Santilli showed that the isotopies of the rotational symmetry $S O(3)$ characterized by isotopic element (27) do represent extended, generally non-spherical and deformable bodies. Finally, Santilli proved in Vol. II of Ref. [1] the significance of his Lie-isotopic theory by showing that it characterizes the Birkhoffian covering of classical Hamiltonian mechanics and its "direct universality" for the representation of all possible, non-singular, generally non-Hamiltonian Newtonian systems in the frame of the experimenter, which direct universality was subsequently proved to hold also for isotopic operator theories. The above advances were formulated on an ordinary numeric field.

Subsequently, Santilli discovered in 1993 [2] that the axioms of numeric fields with characteristic zero do not necessarily require that the basic multiplicative unit is the trivial number +1 , since said axioms admit arbitrary generalized units, today called Santilli isounits, provided that they are positive-definite and are the inverse of the isotopic element, $\hat{I}=1 / \hat{T}>0$. This second historical discovery identified new numbers today known as Santilli isoreal, isocomplex and isoquaternionic numbers of the First (Second) kind when the isounit is outside (an element of) the original field. This discovery prompted a flurry of reformulation over Santilli isofields of all preceding isotopies, including most importantly the reformulation of Santilli's Lie-isotopic theory.

Despite the above momentous advances, Santilli remained dissatisfied because the isotopic formulations of the early 1990s were not invariant under their time evolution, thus being unable to predict the same numerical values under the same conditions at different times. Since the entire 20th century mathematics had been isotonically lifted by the early 1990s, Santilli was left with no other choice than that of reinspecting the Newton-Leibnitz differential calculus by discovering that, contrary to a popular belief in mathematics and physics for some four centuries, the differential calculus is indeed dependent on the basic multiplicative unit. In this way, Santilli achieved in memoir [3] of 1996 the third historical discovery according to which the ordinary differential calculus needs generalizations of the type (28), (29) whenever the isounit depends on the local variable of differentiation. This discovery signaled the achievement of mathematical maturity of isomathematics that permitted numerous advances in physics and chemistry as well as novel industrial applications.

All in all, Santilli has written about 150 papers on the isotopies of all various aspects of 20th century mathematics. These contributions are reported in monographs [4] of 1995 that remain to this day the most comprehensive presentation on isotopies. In the subsequent series of monographs [5] of 2008, Santilli introduces the names of Hadronic Mathematics, Mechanics and Chemistry which have been adopted for this review due to their wide acceptance.
Numerous authors have made important contributions in Santilli isomathematics, among whom we quote: the mathematician H. C. Myung who initiated (with R. M. Santilli) [6] the isotopies of Hilbert Spaces, including the momentous elimination of the divergencies of quantum mechanics under sufficiently small values of the isotopic element $\hat{T}$; the mathematicians D. S. Sourlas and G. T. Tsagas [7] who conducted in 1993 the first comprehensive study of the Lie-Santilli isotheory; the theoretician J. V. Kadeisvili [8] who presented systematic studies of Santilli's isotopies of 20th century geometries and relativities; the mathematician Chun-Xuan Jiang [9] who conducted in 2001 systematic studies of Santilli IsoNumber Theory; the mathematicians R. M. Falcon Ganfornina and J. Nunez Valdes who wrote in 2001 the now historical, first mathematically rigorous treatment of Santilli isotopies [10], and the historical achieved isotopology [11] which provides the ultimate mathematical structure of the Newton-Santilli isoequations (42) for extended-deformable particles under Hamiltonian and non-hamiltonian interactions achieved in memoir [3]; the mathematician S. Georgiev who wrote one of the most monumental and important mathematical works in scientific history [12], by showing that Santilli's IsoDifferential Calculus implies a variety of fully consistent coverings of 20th century mathematics; the mathematician A. S. Muktibodh [13] who presented the first known generalization of Santilli isonumber theory for the case of characteristic $p \neq 0$; the physicists I. Gandzha and J. Kadeisvili who presented in 2011 [14] a comprehensive review of Santilli isomathematics and its applications in physics and chemistry; plus additional seminal advances presented in the subsequent papers of this collection.

\section{Genomathematics, Genomechanics and Genochemistry}

\subsection{Represented Systems s [1-5]}

Single-valued, time-irreversible system of extended-deformable particles under action at a distance Hamiltonian and contact non-Hamiltonian interactions, as occurring in nuclear reactions, biological structures and chemical reactions.

\subsection{Main Mathematical Structure s [1-5]}

Santilli Forward GenoUnit

$$
\hat{I}^{>}=\hat{I}^{>}\left(t^{>} r^{>}, p^{>}, a^{>}, \psi^{>}, \partial^{>} \psi^{>}, \ldots\right)=1 / \hat{T}^{>}>0,(51)
$$

Santilli Backward GenoUnit

$$
<\hat{I}={ }^{<} \hat{I}\left({ }^{<} r,{ }^{<} p,{ }^{<} a,{ }^{<} \psi,{ }^{<}<\psi, \ldots\right)=1 /<\hat{T}>0,
$$

Condition for time-irreversibility

$$
\hat{I}^{>} \neq<\hat{I}
$$

Forward GenoFields 


$$
\hat{F}^{>}\left(\hat{n}^{>},>, \hat{I}^{>}\right), \hat{n}^{>}=n \times \hat{I}^{>}
$$

Backward GenoFields

$$
<\hat{F}(<\hat{n},<,<\hat{I}), \quad<\hat{n}=<\hat{I} \times n,
$$

Forward GenoProduct

$$
\begin{gathered}
\hat{n}>\hat{m}=\hat{n}^{>} \times \hat{T}^{>} \times \hat{m}^{>} \in \hat{F}^{>}, \\
\hat{I}^{>}>\hat{n}^{>}=\hat{n}^{>}>\hat{I}^{>}=\hat{n}^{>} \forall \hat{n}^{>} \in \hat{F}^{>}
\end{gathered}
$$

Backward Genoproduct

$$
\begin{gathered}
<\hat{n}<^{<} \hat{m}=^{<} \hat{n} x^{<} \hat{T} x^{<} \hat{m} \epsilon^{<} \hat{F}, \\
<\hat{I}<<\hat{n}=<\hat{n}<^{<} \hat{I}=<\hat{n} \forall \quad<\hat{n} \epsilon^{<} \hat{F},
\end{gathered}
$$

Representation of forward extended-deformable particles under non-Hamiltonian interactions

$$
\hat{T}^{>}=\operatorname{Diag} \cdot\left(\frac{1}{n_{1}^{2}}, \frac{1}{n_{2}^{2}}, \frac{1}{n_{3}^{2}}\right)^{>} \times e^{\Gamma(t, r, p, \psi, \partial \psi, \ldots)^{>}}
$$

Forward GenoCoordinates

$$
\hat{r}^{>}=r \times \hat{I}^{>} \in \hat{F}^{>},
$$

Backward GenoCoordinates

$$
<\hat{r}=<\hat{I} \times r \epsilon^{<} \hat{F},
$$

Forward GenoFunctional analysis

$$
\hat{f}^{>}\left(\hat{r}^{>}\right)=f\left(\hat{r}^{>}\right) \times \hat{I}^{>} \in \hat{F}^{>},
$$

Backward GenoFunctional analysis

$$
<\hat{f}(<\hat{r})=f(<\hat{r}) x^{<} \hat{I} \epsilon^{<} \hat{F},
$$

Forward GenoDifferential Calculus

$$
\begin{gathered}
\hat{d}^{>} \hat{r}^{>}=d r+r \times \hat{T}^{>} \times d \hat{I}^{>}, \\
\frac{\hat{\partial}^{>} \hat{f}^{>}\left(\hat{r}^{>}\right)}{\hat{\partial} \hat{r}^{>}}=\hat{I}^{>} \times \frac{\partial \hat{f}^{>}\left(\hat{r}^{>}\right)}{\left.\partial \hat{r}^{>}\right)},
\end{gathered}
$$

Backward GenoDifferential Calculus

$$
\begin{gathered}
<\hat{d}^{<} \hat{r}=d r+r \times<\hat{T} \times d^{<} \hat{I}, \\
\frac{<\hat{\partial}<\hat{f}(\hat{r})}{<\hat{\partial}<\hat{r}}=<\hat{I} \times \frac{\partial^{<} \hat{f}(\hat{r})}{\partial<\hat{r}},
\end{gathered}
$$

Santilli Lie-Admissible Theory

$$
\begin{gathered}
\left(X_{i}^{\hat{}}, X_{j}\right)=X_{i}<X_{j}-X_{j}>X_{i}=C_{i j}^{k}(t, r, p, \psi, \ldots) \times X_{k}, \\
A(w)=\hat{e}_{>}^{X \times w \times i}>A(0)<_{<} \hat{e}^{-i \times w \times X} .
\end{gathered}
$$

Santilli Forward Geno-Euclidean Geometry

$$
\begin{gathered}
\hat{E}^{>}\left(\hat{r}^{>}, \hat{\delta}^{>}, \hat{I}^{>}\right), \hat{\delta}^{>}(t, r, p, \psi, \ldots)=\hat{T}^{>}(t, r, p, \psi, \ldots) \times \delta, \\
\hat{r}^{>\widehat{2}}=\hat{r}^{>i}>\hat{\delta}_{i j}^{>}>\hat{r}^{>j} \in F^{>}, \\
\hat{\delta}^{>} \neq \hat{\delta}^{>\text {tranp }}
\end{gathered}
$$

\section{Santilli Backward Geno-Euclidean Geometry}

$$
\begin{aligned}
& <\hat{E}\left(<\hat{r}^{<} \hat{\delta},<\hat{I}\right), \quad<\hat{\delta}(t, r, p, \psi, \ldots)=<\hat{T}(t, r, p, \psi, \ldots) \times \\
& { }^{<2} \hat{r}={ }^{<i} \hat{r}<\hat{\delta}_{i j}<^{<j} \hat{r} \in^{<} F, \\
& <\hat{\delta} \neq<\text { transp } \hat{\delta}
\end{aligned}
$$

Santilli Forward Geno-Minkowskian Geometry $(\mu=$ $1,2,3,4)$

$$
\begin{gathered}
\widehat{M}^{>}\left(\hat{x}^{>}, \hat{\eta}^{>}, \hat{I}^{>}\right): \hat{x}^{>}=\left(\hat{x}^{>\mu}\right), x_{4}^{>}=t^{>}, \\
\hat{\eta}^{>}(x, \psi, \ldots)=\hat{T}^{>}(x, \psi, \ldots) \times \eta, \\
\hat{x}^{>2}=\hat{x}^{>\mu}>\hat{\eta}_{\mu \nu}^{>}>\hat{x}^{>} \in \hat{F}^{>}, \\
\hat{\eta}^{>} \neq \hat{\eta}^{>\text {trans } p}
\end{gathered}
$$

Santilli Backward Geno-Minkowskian Geometry $(\mu=$ $1,2,3,4$,

$$
\begin{gathered}
<\widehat{M}\left({ }^{<} \hat{x},{ }^{<} \hat{\eta},<\hat{I}\right): \quad<\hat{x}=\left(\hat{x}^{\mu}\right), \quad<x_{4}=<t, \\
<\hat{\eta}(x, v, \ldots)=<\hat{T}(x, v, \ldots) \times \eta, \\
<\hat{x}^{<2}=<\mu \hat{x}<{ }^{<} \hat{\eta}_{\mu \nu} \quad<{ }^{<v} \hat{x} \in \epsilon^{<} \hat{F}, \\
<\hat{\eta} \neq{ }^{<\text {transp }} \hat{\eta}
\end{gathered}
$$

Santilli Forward Geno-Riemannian Geometry

$$
\begin{gathered}
\hat{R}^{>}\left(\hat{x}^{>}, \hat{g}^{>}, \hat{I}^{>}\right): \hat{g}^{>}=\hat{T}^{>}(x, v, \ldots) \times g(x), \\
\hat{x}^{>2}=\hat{x}^{>\mu}>\hat{g}_{\mu \nu}^{>}>\hat{x}^{>} \in \hat{F}^{>}, \\
\hat{g}^{>} \neq \hat{g}^{>\text {transp }}
\end{gathered}
$$

Santilli Backward Geno-Riemannian Geometry

$$
\begin{gathered}
<\hat{R}(<\hat{x},<\hat{g},<\hat{I}): \quad<\hat{g}=<\hat{T}(x, v, \ldots) \times g(x), \\
<\hat{x}^{<2}=<\mu \hat{x}<\hat{g}_{\mu \nu} \quad<v \hat{x} \in \in^{<} \hat{F} \\
<\hat{g} \neq{ }^{<\text {transp }} \hat{g}
\end{gathered}
$$

Santilli Forward Geno-Symplectic Geometry

$$
\widehat{\omega}^{>}=\hat{d}^{>} \hat{r}^{>k} \hat{\Lambda}^{>} \hat{d}^{>} \hat{p}_{k}^{>}
$$

Santilli Backward Geno-Symplectic Geometry

$$
<\widehat{\omega}=<\hat{d}^{<} \hat{r}^{<} \hat{\Lambda}^{<} \hat{d}^{<} \hat{p}_{k}
$$

\subsection{GenoDynamical GenoEquations s [1-5]}

Newton-Santilli Forward GenoEquation

$$
\begin{aligned}
\hat{m}^{>}>\frac{\hat{d}^{>} \hat{v}^{>}}{\hat{d}^{>} t^{>}}-F^{>S A}(t, r, p)= & {\left[m \times \frac{d v}{d t}\right]^{>}-F^{S A>}(t, r, p)-} \\
& F^{N S A>}(t, r, p, \ldots)=0,(93)
\end{aligned}
$$

Newton-Santilli Backward GenoEquation

$$
<\widehat{m}<\frac{<\hat{d}^{<} \hat{v}}{<\hat{d}^{<} \hat{t}}-<S A F(t, r, p)=
$$




$$
={ }^{<}\left[m \times \frac{d v}{d t}\right]-{ }^{<S A} F(t, r, p)-{ }^{<N S A} F(t, r, p, \ldots)=0,
$$

Forward GenoVariational principle

$$
\hat{\delta}^{>} \hat{A}^{>}=\hat{\delta}^{>} \widehat{\int}\left(\hat{p}_{k}^{>}>\hat{d}^{>} \hat{r}^{>k}-\widehat{H}^{>}>\hat{d}^{>} \hat{t}^{>}\right)=0 .
$$

Backward GenoVariational principle

$$
{ }^{<} \hat{\delta}^{<} \hat{A}=<\hat{\delta}^{<} \widehat{\int}\left({ }^{<} \hat{p}_{k}<<\hat{d}^{<} \hat{r}^{k}-<\widehat{H}<\hat{d}^{<} \hat{t}\right)=0 .
$$

Forward Hamilton-Santilli GenoEquations

$$
\left[\frac{\hat{d} \hat{r}^{k}}{\hat{d} \hat{t}}\right]^{>}=\left[\frac{\widehat{\partial} \hat{H}(\hat{r}, \hat{p})}{\widehat{\partial} \hat{p}_{k}}\right]^{>},\left[\frac{\widehat{d} \hat{p}_{k}}{\hat{d} \hat{t}}\right]^{>}=-\left[\frac{\widehat{\partial} \widehat{H}(\hat{r}, \hat{p})}{\widehat{\partial} \hat{r}^{k}}\right]^{>},
$$

Backward Hamilton-Santilli GenoEquations

$$
<\left[\frac{\hat{d} \hat{r}^{k}}{\hat{d} \hat{t}}=\frac{\widehat{\partial} \widehat{H}(\hat{r}, \hat{p})}{\widehat{\partial} \hat{p}_{k}}\right], \quad<\left[\frac{\hat{d} \hat{p}_{k}}{\hat{d} \hat{t}}\right]=-<\left[\frac{\widehat{\partial} \widehat{H}(\hat{r}, \hat{p})}{\widehat{\partial} \hat{r}^{k}}\right],
$$

Forward Geno-Hilbert space $\widehat{H}^{>}$with states $\mid \hat{\psi}^{>}>$over the isofield $\hat{C}^{>}$

GenoExpectation value of a Hermitean operator $\hat{A}$ on $\widehat{H}^{>}$

$$
<\hat{A}^{>}>=<^{<} \hat{\psi}\left|<\hat{A}^{>}>\right| \hat{\psi}^{>}>\in \hat{C}
$$

Heisenberg-Santilli GenoEquation ${ }^{3}$

$$
\begin{array}{r}
\hat{\imath} \times \frac{\hat{d} \hat{A}}{\hat{d} \hat{t}}=(\hat{A}, \widehat{H})=\hat{A}<\widehat{H}-\widehat{H}>\hat{A}=A \times<(\hat{\psi}, \ldots) \times \\
\widehat{H}(\hat{r}, \hat{p})-\widehat{H}(\hat{r}, \hat{p}) \times \hat{T}^{>}(\hat{\psi}, \ldots) \times \hat{A}(100)
\end{array}
$$

Forward Schrödinger-Santilli GenoEquation

$$
\begin{array}{r}
\widehat{H}^{>}>\left|\hat{\psi}^{>}>=\widehat{H}^{>}(\hat{r}, \hat{p}) \times \hat{T}^{>}(\hat{\psi}, \hat{\partial} \hat{\psi}, \ldots) \times\right| \hat{\psi}^{>}>=\hat{E}^{>}> \\
\left|\hat{\psi}^{>}>=E^{>} \times\right| \hat{\psi}^{>}>,(101) \\
\hat{p}^{>}>\left|\hat{\psi}^{>}>=-\hat{\imath}^{>}>\hat{\partial}_{\hat{r}}^{>}\right| \hat{\psi}^{>}>=-i \times \hat{I}^{>} \times \partial_{\hat{r}} \mid \hat{\psi}^{>}>,(102)
\end{array}
$$

Backward Schrödinger-Santilli GenoEquation

$$
\begin{array}{r}
<^{<} \hat{\psi}\left|<^{<} \widehat{H}=<^{<} \hat{\psi}\right| x^{<} \hat{T}(\hat{\psi}, \hat{\partial} \hat{\psi}, \ldots) x^{<} \hat{H}(\hat{r}, \hat{p})= \\
<^{<} \hat{\psi}\left|<^{<} \hat{E}=<^{<} \hat{\psi}\right| x^{<} E,(103) \\
<^{<} \hat{\psi}\left|<^{<} \hat{p}=-<^{<} \hat{\psi}\right|<^{<} \hat{\imath}<_{\hat{r}}^{<} \hat{\partial}=-i \times<\left.^{<} \hat{\psi}\right|_{\hat{r}} ^{<} \partial x^{<} \hat{I}
\end{array}
$$

Forward Dirac-Santilli IsoEquation

$$
\begin{array}{r}
\left(\hat{\eta}^{>\mu \nu}>\hat{\gamma}_{\mu}^{>}>\hat{p}_{v}^{>}-\hat{\imath}^{>}>\hat{m}^{>}>\hat{c}^{>}\right)>\mid \hat{p} s i^{>}>=0 . \\
\left\{\hat{\gamma}_{\mu}, \hat{\gamma}_{v}\right\}^{>}=\left[\hat{\gamma}_{\mu} \widehat{\times} \hat{\gamma}_{v}+\hat{\gamma}_{v} \hat{\times} \hat{\gamma}_{\mu}\right]^{>}=\hat{2}^{>}>\hat{\eta}_{\mu v}^{>},
\end{array}
$$

Backward Dirac-Santilli GenoEquation

$$
<^{<} \hat{\psi} \mid<\left({ }^{<} \hat{p}_{v}<^{<} \hat{\gamma}_{\mu}<^{<\mu \nu} \hat{\eta}-{ }^{<} \hat{\imath}<\widehat{m}<^{<} \hat{c}\right)=0 .
$$

\footnotetext{
${ }^{3}$ By including the multi-valued (Section 4) and hyperstructural formulations (Section 5), Lie-admissible equations (100) are so broad that it will take centuries for their generalizations. For this reason,Santilli has requested in his will that his tombstone should have the engraving

$$
i \dot{A}=A<H-H>A
$$

below his name.
}

$$
\begin{aligned}
<\left\{\hat{\gamma}_{\mu}, \hat{\gamma}_{v}\right\}=<\left[\hat{\gamma}_{\mu} \widehat{\times} \hat{\gamma}_{v}+\hat{\gamma}_{v} \widehat{\times} \hat{\gamma}_{\mu}\right] & =<\hat{2}<^{<} \hat{\eta}_{\mu \nu}= \\
2 & \times<\hat{\eta}_{\mu \nu},(108)
\end{aligned}
$$

\subsection{Comments and References}

As it is also well known, all 20th century mathematical, physical or chemical formulations are reversible over time. Following research over half a century initiated during his $\mathrm{Ph}$. D. studies at the University of Torino, Italy, in the mid 1960s $[15,17-23,4,5]$, R. M., Santilli has made the additional historical discovery of the first and only known, axiomatically consistent, generalization of 20 th century mathematics as well as of its covering isomathematcs into a form embedding irreversibility over time in ordered forward and backward units, in corresponding ordered forward and backward products and, consequently, in all subsequent mathematical structures, resulting in the new mathematics nowadays known as Santilli forward and backward genomathematics with corresponding physical and chemical theories for the representation of irreversible processes.

Since the reversibility over time of 20th century theories can be reduced to the invariance under anti-Hermiticity of the Lie product between Hermitean operators, $[a, b]=a b-$ $b a=-[a, b]^{\dagger}$, Santilli presented in 1967 [15] the first known (p, q)-deformation of the Lie product $(a, b)=p a b-q b a$, where $\mathrm{p}, \mathrm{q}$ are scalars and the product $a b$ is generally non-associative. Following an intense search in European mathematical libraries, Santilli discovered that the new product verifies the axiom of Lie-admissibility by the American mathematician A, A, Albert [16] in the sense that the attached anti-symmetric product $[a, b]=(a, b)-$ $(b, a)$ verifies the axioms of a Lie algebra.

Since spaceship during re-entry are notoriously irreversible over time, Santilli was invited by the Center for Theoretical Physics of the University of Miami, Florida, under NASA support, where he moved with his wife Carla and newly born daughter Luisa inAugust 1967, and published a number of additional works in Lie-admissibility, including the first known Lie-admissible generalization of Hamilton and Heisenberg equations [17,18], nowadays considered at the foundation of hadronic mechanics and chemistry, as well as the first and only known Lie-admissible formulation of dissipative plasmas surrounding spaceships during reentry [19].

Santilli then spent seven years, from 1968 to 1974 , at the Department of Physics of Boston University, and then three years, from 1974 to 1977, at MIT, during which tine he wrote, in his words, Phys.. Rev of career-oriented papers nobody reads. InSeptember 1977, Santilli joined Harvard University and was invited by the DOE to study irreversible processes because all energy releasing processes are irreversible over time. In April 1978, Santilli published under his DOE support his most important mathematical contribution [20] (see also monographs [21]) in which he achieved a Lie-admissible covering of the various branches of Lie's theory, Eqs. (69), (70), including the most general known time evolution whose brackets characterize an algebra, Eqs. (1000). It should be indicated that the isotopies of Lie's theory outlined in the preceding section were derived by Santilli as a particular case of the broader Lie-admissible theory of Ref. [20], and then 
published in monographs [1].

Subsequently, Santilli discovered in paper [2] of 1993 that the axiom of a numeric field, besides admitting a generalization of the multiplicative unit, also admit the restriction of the associative product to an ordered form to the right and, separately,to the left. In this way, Santilli discovered two additional classes of new numbers, today known as Santilli forward and backward genoreal, genocomplex and genoquarternionic numbers. In the seminal memoir [3] of 1996 Santilli discovered two additional coverings of the ordinary differential calculus and of its isotopic covering, today known as Santilli forward and backward genodifferential calculi, Eqs. (65) to (68). Santilli called a genotopy [20] the lifting of isomathematics into ordered formulations to the right and to the left in the Greek sense of inducing a covering of Lie's axioms, Eqs. (69), (70).

As it is well known, thousands of papers have been published beginning from the late 1980 s on the so-called q-deformations of Lie algebras with product $(a, b)=a b-$ $q b a$ which are an evident particular case of Santilli Lie-admissible product [15]. Whatit is lesser known, or not admitted, all q-deformations did not achieve invariance over time, thus being afflicted by serious inconsistencies, since they consisted of non-unitary theories formulated via the mathematics of unitary theories. Santilli solved this problem in 1997 by achieving the first and only known invariant formulation of q-as well as of (p, q)-deformations [22].

We should indicate that Santilli's conception of a genotopic lifting of his preceding isomathematcs (indicated in Section 2 by "hat" on symbols plus the "arrow of time") is necessary to achieve a consistent representation of irreversibility because point-like particles can only experience action-at-a-distance interactions that are reversible over time. Therefore, a simple genotopy of 20th century mathematics based on the conventional associative product would be axiomatically inconsistent. Consequently, to represent irreversibility it is first necessary to lift 20th century mathematics into isomathematcs, with consequential representation of extended-deformable particles via realizations of type (27) so that extended particles can experience non-Hamiltonian interactions needed for irreversibility. It is then necessary to add irreversibility via the ordering of all products. It should also be indicated that, when formulated via time-dependent isounits, isomathematics can becomes genomathematics via the identifications $\hat{I}(t, \ldots)=\hat{I}^{\dagger}(t, \ldots)=\hat{I}^{>}, \hat{I}(-t, \ldots)=$ $\hat{I}^{\dagger}(-t, \ldots)=<\hat{I}, \hat{I}(t, \ldots) \neq \hat{I}(-t)$, and the judicious addition of ordered products.

Systematic studies on the Lie-Admissible treatment of irreversible systems were presented in memoir [3] and monographs [4]. Santilli's subsequent memoir [23] of 2006 remains to this day the most comprehensive presentation of Lie-admissible treatments of irreversibility at the classical and operator levels. Monographs [5] of 2008 presented an update. Paper collection [24[ presents all available independent contributions in Lie-admissibility up to [1984. The Proceedings of the Third International Conference on Lie-admissible Treatment of Irreversible Systems [25] present numerous additional independent contributions as well as references for the five Workshops on Lie-Oadmissible
Algebras organized by Santilli at Harvard University, and for the preceding two international conference in Lie-admissibility, the first at the Université d'Orleans, France, in 1981 and the second at the Castle Prince Pignatelli, Italy, in 1995 (see also the general review [14] and large literature quoted therein).

As it is well known, there exists a large number of papers on Lie-admissible algebras within the context of non-associative algebras (see Tomber's Bibliography [26] listing all significant papers in the field up to 1986). It should be indicated that, regrettably, these studies have no connection with Santilli genomathematics since the latter deals with the irreversible generalizations of all aspects of 20th century mathematics.

\section{Classical Hypermathemates, Hypermechanics and Hyperchemistry}

\subsection{Represented Systems s [1-5]}

Multi-valued, time-irreversible systems of extended -deformable particles or constituents under the most general known Hamiltonian and non-Hamiltonian interaction, as occurring for multi-valued universes or the structure of the DNA.

\subsection{Main Mathematical Structure s [1-5]}

Basic HyperUnits and HyperProducts

$$
\begin{gathered}
\hat{I}^{>}=\left\{\hat{I}_{1}^{>}, \hat{I}_{2}^{>}, \hat{I}_{3}^{>}, \ldots\right\}=1 / \hat{S}, \\
<\hat{I}=\left\{<\hat{I}_{1},<\hat{I}_{2},<\hat{I}_{3}, \ldots\right\}=\frac{1}{\hat{R}^{\prime}}
\end{gathered}
$$

Forward and Backward HyperProducts

$$
\begin{gathered}
A>B=\left\{A \times \hat{S}_{1} \times B, A \times \hat{S}_{2} \times B, A \times \hat{S}_{3} \times B, \ldots\right\}, \hat{I}^{>}> \\
A=A>\hat{I}^{>}=A \times I, \\
A<B=\left\{A \times \hat{R}_{1} \times B, A \times \text { hat } R_{2} \times B, A \times \hat{R}_{3} \times B, \ldots\right\}<\hat{I}< \\
A=-A<^{<} \hat{I}=I \times A, \\
A=A^{\dagger}, B=B^{\dagger}, \hat{R}=\hat{S}^{\dagger} .
\end{gathered}
$$

Classical hypermathematcs then follow as for genomathematcs with multi-valued units, quantities and operations.

\subsection{Classical Hyper-Dynamical Equations s [1-5]}

The same as those for genomathematics, but with multi-valued hyperunits, quantities and operations.

Comments and References

The multi-valued three-dimensional (rather than multi-dimensional) realization of genomathematics outlined in Section 4 emerged from specific biological needs. The Australian biologist C. Illert [27] confirmed that the shape of seashells can indeed be represented in a three-0dimensional Euclidean space as known since Fourier's time, but proved that the growth in time of a seashell cannot any longer be 
consistently represented in a conventional, three-dimensional Euclidean space, and achieved a consistent representation via the doubling of the three reference axis.

Santilli [27,28] confirmed Illert's findings because the conventional Euclidean geometry has no time arrow and, consequently, cannot consistently represent a strictly irreversible system, such as the growth of seashells. Additionally, Santilli proved thathis geno-Euclidean geometry, Eqs. (71) to (73), is equally unable to represent the growth in time of seashells despite its irreversible structure, however, an axiomatically consistent and exact representation of the growth of seashells was possible via the multi-valued realization of the forward geno-Euclidean geometry, thus beginning to illustrate the complexity of biological structures.

The multi-valued, rather than multi-dimensional character of classical hypermathematics is indicated by Santilli as follows [28] We perceive the growth of a seashell specifically in three dimensions from our Eustachian lobes. Therefore, an irreversible mathematics suitable to represent the growth of sea shells must be perceived by us as being in three dimensions. However, Illert has shown the need to double the three Cartesian axis. Classical hypermathematics has been conceived and structured in such a way that the increase of the reference axes is complemented by a corresponding multi-valued hyperunit in such a way that a classical hyper-Euclidean geometry, when seen at the abstract level, remains indeed three-dimensional as necessary to achieve representation of biological structures compatible with our sensory perception.

\section{Hope Hypermathematics, Hypermechanics and Hyperchemistry}

\subsection{Represented Systems}

The most complex known multi-valued, time-irreversible requiring extremely large number of data, such as the DNA code [31-35].

\subsection{Comments and References}

Despite the preceding structural generalization of 20th century mathematics, Santilli remained dissatisfied in view of the complexity of nature, particularly of biological entities because advances in the structure of the DNA are indeed possible via classical hypermathematics, as we shall see in the third collection of this series dedicated to chemistry (e.g., via Santilli hypermagnecules), but any attempt at representing the DNA code via any of the preceding mathematics can be proved to be excessively restrictive due to the volume, complexity, diversification and coordination of the information.

Therefore, Santilli approved one of the most important mathematicians in hyperstructures, T. Vougiouklis from Greece, and asked for his assistance in further generalizing the preceding mathematics via hyperstructures defined on hyperfields, as necessary for applications implying measurements, and formulated via hyperoperations (called "hope") permitting the needed broadening of the representational capability.

The above contact lead to the hypermathematics indicated in this section as presented in Refs. [29-33] which is based on Vougiouklis $H_{v}$ hyperaxioms and which mathematics, in Santilli's words, constitutes the most general mathematics that can be conceived nowadays by the human mind.

\section{Isodual Mathematics, Mechanics and Chemistry}

\subsection{Represented Systems}

Single-valued, closed-isolated, time-reversible systems of classical and operatorpoint-like antiparticles moving in vacuum solely under action at a distance Hamiltonian interactions, such as the stricture of antimatter atoms and antimatter molecules [2,36-43].

\subsection{Main Mathematical Structure [2, 36-43]}

Basic isodual unit

$$
1^{d}=-1^{\dagger}=-1
$$

Isodual numeric fields

$$
\begin{aligned}
F^{d}\left(n^{d}, \times^{d}, 1^{d}\right), n^{d}=n \times 1^{d}, n^{d} & \times^{d} m^{d} \\
& =n^{d} \times\left(1^{d}\right)_{-1} \times m^{d} \in F^{d},
\end{aligned}
$$

$n^{d}=$ isodual! real,complex, quatern.! numbers,

Isodual functional analysis

$$
f^{d}\left(r^{d}\right)=f\left(r^{d}\right) \times 1^{d} \in F^{d}
$$

Isodual differential calculus

$$
\begin{gathered}
d^{d} r^{d}=(1)^{-1} \times d r^{d}=d r, \\
\frac{\partial^{d} f^{d}\left(r^{d}\right)}{\partial^{d} r^{d}}=1^{d} \times \frac{\partial f^{d}\left(r^{d}\right)}{\partial r^{d}},
\end{gathered}
$$

Santilli Isodual Lie theory

$$
\begin{gathered}
{\left[X_{i}, X_{j}\right]^{d}=\left(X_{i} \times X_{j}-X_{j} \times X_{i}\right)^{d}==-C_{i j}^{k} \times X_{k},} \\
A^{d}\left(w^{d}\right)=e_{d}^{X \times w \times i} \times^{d} A^{d}(0) \times^{d} e_{d}^{-i \times w \times X} .
\end{gathered}
$$

Santilli isodual Euclidean geometry

$$
\begin{gathered}
E^{d}\left({ }^{d}, \delta^{d}, 1^{d}\right), r^{d}=\left(r^{d k}\right), k=1,2,3, \\
\delta^{d}=\operatorname{Diag} \cdot(-1,-1,-1), \\
r^{d 2 d}=r^{d i} \times \delta_{i j} \times^{d} r^{d j}=\left(r_{1}^{2}+r_{2}^{2}+r_{3}^{2}\right) \times 1^{d} \in F^{d},
\end{gathered}
$$

Santilli Isodual Minkowskian geometry $(\mu=1,2,3,4$,

$$
\begin{gathered}
M^{d}\left(x^{d}, \eta^{d}, I^{d}\right): x^{d}=\left(x^{d \mu}\right), x^{d 4}=t^{d}=t \times 1^{d}=-t \\
\eta^{d}=\text { Diag. }\left(-1,-1,-1,+c^{d 2 d}\right)
\end{gathered}
$$




$$
\begin{aligned}
x^{d 2 d} & =\left(x^{\mu} \times \eta_{\mu \nu} \times x^{\nu}\right)^{d} \\
& =\left(x_{1}^{2}+x_{2}^{2}+x_{3}^{2}-t^{2} c^{2}\right) \times 1^{d} \in F^{d},
\end{aligned}
$$

Isodual Riemannian geometry, Santilli Isodual Symplectic Geometry.

\subsection{Isodual Dynamical Equations [2, 36-43]}

Newton-Santilli Isodual Equation

$$
m^{d} \times^{d} \frac{d^{d} v^{d}}{d^{d} t^{d}}-F^{d S A}\left(t^{d}, r^{d}, v^{d}\right)=0,
$$

Isodual Variational Principle

$$
\delta^{d} A^{d}=\delta^{d} \int^{d}\left(p_{k}^{d} \times^{d} d^{d} r^{d k}-H^{d} \times^{d} d^{d} t^{d}\right)=0 .
$$

Hamilton-Santilli Isodual Equations without external terms

$$
\frac{d^{d} r^{d k}}{d^{d} t^{d}}=\frac{\partial^{d} H^{d}\left(r^{d}, p^{d}\right)}{\partial^{d} p_{k}^{d}}, \frac{d^{d} p_{k}^{d}}{d^{d} t^{d}}=-\frac{\partial^{d} H^{d}\left(r^{d}, p^{d}\right)}{\partial^{d} r^{d k}},
$$

Isodual Hilbert space $H^{d}$ over $C$ with states $\left|\psi^{d}\right\rangle=-<$ $\psi \mid$ over $C^{d}$

Expectation value of a Hermitean operator $A$

$$
<A^{d}>=<\psi\left|\times A^{d} \times\right| \psi>\in C^{d} m
$$

Heisenberg-Santilli Isodual Equations

$$
i^{d} \times^{d} \frac{d^{d} A^{d}}{d^{d} t^{d}}=[A, H]^{d}=(A \times H-H \times A)^{d},
$$

Schrödinger-Santilli Isodual Equations

$$
\begin{gathered}
H^{d} \times^{d}\left|\psi^{d}>=E^{d} \times^{d}\right| \psi^{d}>=-E \times \mid \psi> \\
p^{d} \times^{d}\left|\psi^{d}>=+i^{d} \times^{d} \partial_{r d}^{d}\right| \psi^{d}>
\end{gathered}
$$

Dirac-Santilli Isodual Equation

$$
\begin{gathered}
\left(\eta^{d \mu \nu} \times^{d} \gamma_{\mu}^{d} \times^{d} p_{\nu}^{d}+i^{d} \times^{d} m^{d} \times^{d} c^{d}\right) \times \mid \psi>=0 \\
\left\{\gamma_{\mu}, \gamma_{\nu}\right\}^{d}=\left(\gamma_{\mu} \times \gamma_{\nu}+\gamma_{n} u \times \gamma_{\mu}\right)^{d}=2^{d} \times^{d} \eta_{\mu \nu}^{d},
\end{gathered}
$$

Comments and References

In addition to the the study of irreversible processes and the representation of extended-deformable particles, during his $\mathrm{Ph}$ D. studies of the md 1960s Santilli was interested to ascertain whether a far away galaxy is made up of matter or of antimatter. He soon discovered that none of the mathematics and physics he had learned during his graduate studies was applicable for a quantitative study of the problem considered since, at that time, antimatter was solely represented in second quantization, while the study of far away antimatter galaxies requested their representation at the purely classical and neutral level. In this way, Santilli initiated a solitary scientific journey that lasted for half a century.

This occurrence created one of the biggest imbalances in scientific history because matter was treated at all possible levels, from Newtonian mechanics to second quantization, while antimatter was solely treated in second quantization. The imbalance originated from the fact that special and general relativities had been conceived decades before the discovery of antimatter and, therefore, they had no possibility of representing antimatter at the classical and neutral (as well as charged) level.

It should be stressed that the ongoing trend to extend the application of special and general relativities to the classical treatment of antimatter is afflicted by a number of serious inconsistencies, such as the impossibility to achieve a consistent representation of neutral antimatter, the impossibility to reach a consistent representation of matter-antimatter annihilation (evidently due to the lack of a suitable conjugation from matter to antimatter), violation of the PCT theorem and other inconsistencies that remain generally ignored.

Being an applied mathematician by instinct and training, Santilli knew that the imbalance was the result of a purely mathematical insufficiency because the transition from matter to antimatter is an anti-homomorphism. Consequently, the description of antimatter required a mathematics which is anti-homomorphic to conventional mathematics.

Santilli dedicated a decade to the search of the needed mathematics for antimatter. Following an additional extended search done at the Department of Mathematics of Harvard University under DOE support in the early 1980s, Santilli concluded that a mathematics suitable for the joint classical and operator treatment of antimatter did not exist and had to be constructed.

In the early $1980 \mathrm{~s}$, Since he had introduced the isoproduct $A \widehat{\times} B=A \times \widehat{T} \widehat{B}, \widehat{T}>0$, Eq. (25). Consequently, it was natural to introduce its negative-definite counterpart which he called isodual and denoted with theupper index $d$, namely $A \widehat{\times}^{d} B=A \times \widehat{T}^{d} \hat{B}, \widehat{T}^{d}=\left(\widehat{T}^{d}\right)^{\dagger}<0$. While constructing the isotopies of 20th century mathematics presented in Section 2, Santilli initiated the construction of their isodual image but published no paper in the new mathematics for over a decade.

This caution was due to the fact that, despite the lack of any visible mathematical inconsistency, Santilli remained skeptical on a mathematics based on a negative-definite product is afflicted by known physical inconsistencies, such as the violation of causality for negative time, energies and other physical quantities.

A breakthrough occurred in paper [2] of 1993. During the achievement of the broadest possible realizations of the abstract axioms of a numeric field (of characteristic zero), Santilli discovered that realizations with negative-definite units were simply unavoidable. This lead to the discovery of additional new numbers, today known as Santilli isodual real, isodual complex and isodual quaternionic numbers occurring for $I^{d}=-1$, Eq. (14), with isodual products (5), which are at the foundation of the isodual mathematics of this section and the additional numbers known as Santilli isodual iso- and isodual geno-real, complex and quaternionic numbers which are at the foundation of the isodual isomathematics and isodual genomathematics of Sections 7 and $8 \mathrm{~m}$ respectively [2].

The discovery of isodual numbers is truly historical in our view due to its far reaching implications. In fact, the discovery 
established the existence of the desired isodual mathematics as an anti-isomorphic image of 20th century mathematics for the representation of antimatter. Additionally, the discovery permitted the resolution of the problems of causality for negative values of physical quantities.

To avoid insidious inconsistencies generally not seen by non-experts in the field, the isodual map must be applied for consistency to the totality of quantities and their operations. This lead to Santilli's conception of antimatter as possessing it negative-definite physical quantities for time, energy, momentum, frequency, etc, but such negative values are referred to negative units of measurements. Consequential a theory with negative time referred to negative units of time is as causal as our reality with a positive time referred to positive units, and the same holds for all other physical quantities.

Following the resolution of these basic issues, Santilli published in 1994 his first paper [36] specifically devoted to the isodual representation of antimatter. In mathematical memoir [3] of 1996, Santilli achieved the first isodual mathematical and physical representation of antimatter. In paper [37] of 1998, Santilli achieved his first goal of the early 1960s, namely, a consistent classical representation of neutral (as well as charged) antimatter.

By the early 1990s, Santilli had shown that isodual mathematics represents all available experimental, data on antimatter at the classical and operator level. Hence, he initiated the second phase of his studies, namely, the identification of new predictions for subsequent experimental verification.

A breakthrough occurred at the 1996 First International Conference on Antimatter help in Sepino, Italy [38]. By that time, Santilli had shown that the only conceivable representation of neutral antimatter required the conjugation of the sign of all physical quantities (jointly with the corresponding conjugation of their units of measurements). Since photons are neutral, the application of the same principle to light implies light emitted by antimatter, that Santilli called isodual light, is physically different than light emitted by matter in an experimentally verifiable way, e.g., because antimatter light is predicted to be repelled by a matter gravitational field.

Santilli then passed to a deeper geometric study of the gravitational field of antimatter. As indicated earlier, general relativity was formulated decades before the discovery of antimatter and, therefore, had no clue for the representation of the gravitational field of antimatter bodies. In Ref.[39] of 1998, Santilli conducted an in depth geometric study of antimatter, and in monograph [40) of 2006, Santilli completed the gravitational study of antimatter via the isodual Riemannian geometry.

All these studies concluded with the prediction of gravitational repulsion (antigravity) between matter and antimatter at all levels of analysis, from the isodual Newton-Santilli equations (26) to isodual second quantization. These aspects will be studied in the second collection of this series dedicated to hadronic mechanics.

Thanks to all the above advances, Santilli was finally in a position to address his original main aim of the 1960s, namely, ascertain whether a far away galaxy is made up of matter or of antimatter. The preceding studies had established that the light emitted by antimatter must have a negative index of refraction that, as such, require concave lenses for its focusing. Consequently, Santilli secured the construction of a revolutionary telescope with concave lenses. About fifty years following his original aim, Santilli finally published in 2013 [41[ measurements of the night sky with his new telescope showing images that can be solely due to light with a negative index of refraction which light, in turn, can solely originate from far away antimatter stars or galaxies (see also the two independent confirmations [42,43]).

An intriguing aspect that should be of interest to pure mathematicians is the conclusion of these studies illustrating the power of new mathematics, to the effect that none of the large numbers of telescopes available nowadays can detect antimatter starsor galaxies since they all have convex lenses. Similarly, as humans evolved in a matter world, we will never be able to see antimatter with our eyes since our cornea is convex and, as such, it will disperse antimatter light all over the retina.

Needless to say, isodual mathematics and its application to antimatter have implications so intriguing that are stimulating the participation of a large number of scientists as we shall report in the second collection of this series

\section{Isodual Isomathematics, Isodual Isomechanics and Isodual Isochemistry}

\subsection{Represented Systems [2, 36-43]}

Single-value, closed-isolated, time-reversible system of classical or operator extended-deformable antiparticles with action at a distance Hamiltonian and contact non-Hamiltonian interactions, such as the structure of antimatter hadrons, nuclei and stars, in the antimatter valence electron bonds and other antimatter systems.

\subsection{Main Mathematical Structure[2, 36-43]}

Basic Isodual IsoUnit

$$
\hat{I}^{d}=\hat{I}^{d}\left(r^{d}, p^{d}, a^{d}, \psi{ }^{d} \partial^{d} \psi^{d}, \ldots\right)=1^{d} /{ }^{d} \widehat{T}^{d}<0,
$$

Basic Isodual IsoFields

$$
\hat{F}^{d}\left(\hat{n}^{d}, \widehat{x}^{d}, \hat{I}^{d}\right), \hat{n}^{d}=n \times \hat{I}^{d}, \hat{n}^{d} \widehat{\times}^{d} \hat{m}^{d}=\hat{n}^{d} \times \hat{T}^{d} \times \hat{m}^{d} \in
$$

Isodual IsoCoordinates $\hat{r}^{d}=r \times \hat{I}^{d} \in \hat{F}^{d}$, $\widehat{F}^{d}$,

Isodual IsoFunctional analysis $\hat{f}^{d}\left(\hat{r}^{d}\right)==f\left(\hat{r}^{d}\right) \times \hat{I}^{d} \in$ Isodual IsoDifferential Calculus

$$
\begin{gathered}
\hat{d}^{d} \hat{r}^{d}=d r-r^{d} \times \hat{T}^{d} \times d \hat{I}^{d}, \\
\frac{\widehat{\partial}^{d} \hat{f}^{d}(\hat{r})}{\widehat{\partial}^{d} \hat{r}^{d}}=\hat{I}^{d} \times \frac{\partial \hat{f}^{d}\left(\hat{r}^{d}\right)}{\partial \hat{r}^{d}},
\end{gathered}
$$


Santilli Isodual Lie-Isotopic Theory

$$
\begin{aligned}
& \left.\left[X_{i}, X_{j}\right]^{d}=X_{i} \widehat{\times} X_{j}-X_{j} \widehat{\times} X_{i}\right)^{d}==-C_{i j}^{k}(r, p, \ldots) \times X_{k} \\
& A^{d}\left(w^{d}\right)=\hat{e}_{d}^{X^{d} \times w^{d} \times i^{d}} \widehat{x}^{d} A^{d}\left(0^{d}\right) \widehat{x}^{d} \hat{e}_{d}^{-i^{d} \times w^{d} \times X^{d}} .
\end{aligned}
$$

Santilli Isodual Iso-Euclidean Geometry

$$
\begin{array}{r}
\hat{E}^{d}\left(\hat{r}^{d}, \hat{\delta}^{d}, \hat{I}^{d}\right), \hat{\delta}^{d}\left(r^{d}, p^{d}, a^{d}, \psi, \ldots\right)= \\
\hat{T}^{d}\left(r^{d}, p,{ }^{d} a^{d}, \psi^{d}, \ldots\right) \times \delta,(141) \\
\hat{T}^{d}=\operatorname{Diag} \cdot\left(1 / n_{1}^{2}, 1 / n_{2}^{2}, 1 / n_{3}^{2}\right)^{d}, \quad(142)
\end{array}
$$

$\hat{r}^{d \widehat{2} d}=\left(\hat{r}^{i} \widehat{\times} \hat{\delta}_{i j} \widehat{\times} \hat{r}^{j}\right)^{d}=\left(\frac{r_{1}^{2}}{n_{1}^{2}}+\frac{r_{2}^{2}}{n_{2}^{2}}+\frac{r_{3}^{2}}{n_{3}^{2}}\right)^{d} \times \hat{I}^{d} \in \hat{F}^{d}$,

Santilli Isodual Iso-Minkowskian Geometry $(\mu=1,2,3,4)$

$$
\begin{gathered}
\widehat{M}^{d}\left(\hat{x}^{d}, \hat{\eta}^{d}, \hat{I}^{d}\right): \hat{x}^{d}=\left(\hat{x}^{d \mu}\right), \hat{x}_{4}^{d}=\hat{t}^{d}=t \times \hat{I}^{d}, \\
\hat{\eta}^{d}\left(x^{d}, \psi^{d}, \ldots\right)=\hat{T}^{d}\left(x^{d}, \psi^{d}, \ldots\right) \times \eta, \\
\hat{T}^{d}=\operatorname{Diag} .\left(1 / n_{1}^{2}, 1 / n_{2}^{2}, 1 / n_{3}^{2}, 1 / n_{4}^{2}\right)^{d}, \\
\hat{x}^{d \hat{2} d}=\left(\hat{x}^{\mu} \widehat{\times} \hat{\eta}_{\mu \nu} \widehat{\times} \hat{x}^{v}\right)^{d}==\left(\frac{x_{1}^{2}}{n_{1}^{2}}+\frac{x_{2}^{2}}{n_{2}^{2}}+\frac{x_{3}^{2}}{n_{3}^{2}}-t^{2} \frac{c^{2}}{n_{4}^{2}}\right)^{d} \times \\
\hat{I}^{d} \in \hat{F}^{d},(147)
\end{gathered}
$$

Santilli Isodual Iso-Riemannian Geometry

$$
\begin{gathered}
\hat{R}^{d}\left(\hat{x}^{d}, \hat{g}^{d}, \hat{I}^{d}\right): \hat{g}^{d}=\hat{T}^{d}\left(x^{d}, v^{d}, \ldots\right) \times g(x), \\
\hat{x}^{d \widehat{2} d}=\left(\frac{g_{11}}{n_{1}^{2}}+\frac{g_{22}}{n_{2}^{2}}+\frac{g_{33}}{n_{3}^{2}}-\frac{g_{44}}{n_{4}^{2}}\right)^{d} \times \hat{I}^{d} \in \hat{F}^{d},
\end{gathered}
$$

Santilli Isodual Iso-Symplectic Geometry

$$
\widehat{\omega}^{d}=\hat{d} \hat{r}^{d k} \widehat{\Lambda}^{d} \hat{d} \hat{p}_{k}^{d}
$$

\subsection{Isodual IsoDynamical IsoEquation[2,36-43}

Newton-Santilli Isodual IsoEquation

$$
\begin{array}{r}
\hat{m}^{d} \widehat{x}^{d} \frac{\hat{d}^{d} \hat{v}^{d}}{\hat{d}^{d} \hat{t}^{d}}-F^{d S A}\left(r^{d}, p^{d}\right)= \\
\left(m \times \frac{d v}{d t}\right)^{d}-F^{d S A}\left(r^{d}, p^{d}\right)-F^{d N S A}\left(r^{d}, p^{d}, \ldots\right)=0^{d}=0,
\end{array}
$$

Isodual IsoVariational principle

$\hat{\delta}^{d} \hat{A}^{d}=\hat{\delta}^{d} \widehat{\int}^{d}\left(\hat{p}_{k}^{d} \widehat{x}^{d} \hat{d}^{d} h a t r^{d k}-\widehat{H}^{d} \widehat{x}^{d} \hat{d}^{d} \hat{t}^{d}\right)=0^{d}=$

Hamilton-Santilli Isodual IsoEquations

$$
\frac{\hat{d}^{d} \hat{r}^{d k}}{\hat{d}^{d} \hat{t}^{d}}=\frac{\widehat{\partial}^{d} \widehat{H}^{d}\left(\hat{r}^{d}, \hat{p}^{d}\right)}{\widehat{\partial}^{d} \hat{p}_{k}^{d}}, \frac{\hat{d} \hat{p}_{k}}{\hat{d}^{d} \hat{t}^{d}}=+\frac{\widehat{\partial}^{d} \widehat{H}^{d}\left(\hat{r}^{d}, \hat{p}^{d}\right)}{\widehat{\partial}^{d} \hat{r}^{d k}},
$$

Isodual iso-Hilbert space $\widehat{H}^{d}$ over $C$ with states $\left|\hat{\psi}^{d}\right\rangle=$ $-<\widehat{\psi} \mid$ over $\hat{C}^{d}$

Expectation value of a Hermitean operator $A$

$$
<A^{d}>=<\hat{\psi}\left|\widehat{\times} A^{d} \widehat{\times}\right| \hat{\psi}>\in C^{d}
$$

Heisenberg-Santilli Isodual IsoEquation $\hat{\imath}^{d} \widehat{\times}^{d} \hat{d}^{d}$ hat $A^{d}$ over $\hat{d}^{d} \hat{t}^{d}=[\hat{A}, \widehat{H}]^{d}=(\hat{A} \widehat{\times} \widehat{H}-\widehat{H} \widehat{\times} \hat{A})^{d}=$ $(\hat{A} \times \hat{T}(\hat{\psi}, \hat{\partial} \hat{\psi}, \ldots) \times \widehat{H}(\hat{r}, \hat{p})-\widehat{H}(\hat{r}, \hat{p}) \times \widehat{T}(\hat{\psi}, \hat{\partial} \hat{\psi}, \ldots) \times$ $\hat{A})^{d},(155)$

Schrödinger-Santilli Isodual IsoEquation

$(\widehat{H} \widehat{\times} \mid \widehat{\psi}>)^{d}=<\widehat{\psi}^{d} \mid \widehat{x}^{d} \widehat{H}^{d}=(\widehat{H}(\hat{r}, \hat{p}) \times \widehat{T}(\hat{\psi}, \hat{\partial} \hat{\psi}, \ldots) \times$ $\mid \widehat{\psi}>)^{d}=-<\hat{\psi}^{d}\left|\widehat{x}^{d} \hat{E}^{d}=-<\hat{\psi}^{d}\right| \times \hat{E}^{d},(156)$

$(\hat{p} \widehat{\times} \mid \widehat{\psi}>)^{d}=<\hat{\psi}^{d}\left|\widehat{x}^{d} \hat{\partial}_{\hat{r}^{d}}=-i \times<\hat{\psi}^{d}\right| \widehat{x}^{d} \hat{\partial}_{\hat{r}^{d}}^{d}$,

Dirac-Santilli Isodual IsoEquation

$$
\begin{aligned}
& {\left[\left(\hat{\eta}^{\mu \nu} \widehat{\times} \hat{\gamma}_{\mu} \widehat{\times} \hat{p}_{v}-\hat{\imath} \widehat{\times} \hat{m} \widehat{\times} \hat{c}\right) \widehat{\times} \mid \hat{p} s i>\right]^{d}=0 .} \\
& \left\{\hat{\gamma}_{\mu}, \hat{\gamma}_{v}\right\}^{d}=\left(\hat{\gamma}_{\mu} \widehat{\times} \hat{\gamma}_{v}+\hat{\gamma}_{v} \widehat{\times} \hat{\gamma}_{\mu}\right)^{d}=\hat{2}^{d} \widehat{\times}^{d} \hat{\eta}_{\mu \nu}^{d},
\end{aligned}
$$

Comments and References

See monograph [40] with particular reference to the use of the isodual isomathematics for the achievement of a grand unification of electroweak and gravitational interactions inclusive of matter and antimatter.

\section{Isodual Genomathematics, Isodual Genomechanics and Isodual Genochemistry}

\subsection{Represented Systems [2, 36-43]}

Single-valued, time-irreversible system of extended-deformable antiparticles under action at a distance Hamiltonian and contact non-Hamiltonian interactions, as occurring in antimatter nuclear reactions, antimatter biological structures and antimatter chemical reactions.

\subsection{Main Mathematical Structure [2, 36-43]}

Backward Isodual GenoUnit

$$
\hat{I}^{>c}=\hat{I}^{>d}\left(t^{>} r^{>}, p^{>d}, a^{>d}, \psi^{>d}, \partial^{>d} \psi^{>d}, \ldots\right)=1 / \hat{T}^{>d}>
$$

Forward Isodual GenoUnit

$$
{ }^{<d} \hat{I}={ }^{<d} \hat{I}\left({ }^{<d} r,{ }^{<d} p,{ }^{<d} a,{ }^{<d} \psi,{ }^{<d} \partial^{<d} \psi, \ldots\right)=1 /^{<d} \widehat{T}>
$$

Condition for time-irreversibility

$$
\hat{I}^{>d} \neq<d \hat{I}
$$

Backward Isodual GenoFields

$$
\begin{array}{r}
\hat{F}^{>d}\left(\hat{n}^{>d},>, \hat{I}^{>d}\right), \hat{n}^{>d}=n \times \hat{I}^{>d}, \hat{n}^{>d}>^{d} \hat{m}^{>d}=\hat{n}^{>d} \times \\
\hat{T}^{>d} \times \hat{m}^{>d} \in \hat{F}^{>d},(163)
\end{array}
$$

Forward Isodual GenoFields

$$
\begin{array}{r}
<d \hat{F}\left({ }^{<d} \hat{n},<,<d \hat{I}\right), \quad<d \hat{n}=<d \hat{I} \times n, \quad<d \hat{n}<d \quad<d \hat{m}= \\
={ }^{<d} \hat{n} \times{ }^{<d} \hat{T} \times{ }^{<d} \hat{m} \in \in^{<d} \hat{F},(164)
\end{array}
$$

Backward Isodual GenoCoordinates 


$$
\hat{r}^{>d}=r \times \hat{I}^{>d} \in \hat{F}^{>d},
$$

Forward Isodual GenoCoordinates

$$
{ }^{<d} \hat{r}=<d \hat{I} \times r \in \in^{<d} \hat{F},
$$

Backward Isodual GenoFunctional analysis

$$
\hat{f}^{>d}\left(\hat{r}^{>d}\right)=f\left(\hat{r}^{>d}\right) \times \hat{I}^{>d} \in \hat{F}^{>d},
$$

Forward Isodual GenoFunctional analysis

$$
{ }^{<d} \hat{f}\left({ }^{<d} \hat{r}\right)=f\left({ }^{<d} \hat{r}\right) \times{ }^{<d} \hat{I} \in{ }^{d} \hat{F}
$$

Backward Isodual GenoDifferential Calculus

$$
\begin{aligned}
& \hat{d}^{>d} \hat{r}^{>d}=d r+r \times \hat{T}^{>d} \times d \hat{I}^{>d}, \\
& \frac{\hat{\partial}^{>d} \hat{f}^{>d}\left(\hat{r}^{>d}\right)}{\hat{\partial}^{>d} \hat{r}^{>d}}=\hat{I}^{>d} \times \frac{\partial \hat{f}^{>d}\left(\hat{r}^{>d}\right)}{\partial \hat{r}^{>d}},
\end{aligned}
$$

Forward Isodual GenoDifferential Calculus

$$
\begin{aligned}
& <d \hat{d}^{<d} \hat{r}=d r+r \times<d \hat{T} \times d^{<d} \hat{I}, \\
& \frac{<d \hat{\partial}^{<d} \hat{f}\left({ }^{<d} \hat{r}\right)}{<d \hat{\partial}^{<d} \hat{r}}={ }^{<d d} \hat{I} \times \frac{\partial^{<d} \hat{f}\left({ }^{<d} \hat{r}\right)}{\partial^{<d} \hat{r}},
\end{aligned}
$$

Santilli Isodual Lie-Admissible Theory

$$
\begin{array}{r}
\left(X_{i}, X_{j}\right)^{d}=\left(X_{i}<X_{j}-X_{j}>X_{i}\right)^{d}= \\
-C_{i j}^{d k}\left(t^{d}, r^{d}, p^{d}, \psi^{d}, \ldots\right) \times X_{k},(173) \\
A^{d}\left(w^{d}\right)=\hat{e}_{>d}^{X \times w \times i}>^{d} A(0)<_{<d}^{d} \hat{e}^{-i \times w \times X} .(174)
\end{array}
$$

Santilli Backward Geno-Euclidean Geometry

$$
\begin{gathered}
\hat{r}^{>d \widehat{2} d}=\left(\hat{r}^{>d i}>^{d} \hat{\delta}_{i j}^{>d}>\hat{r}^{>d j}=\in F^{>d},\right. \\
\hat{T}^{>d} \neq \hat{T}^{>d \text { transp }}
\end{gathered}
$$$$
\hat{E}^{>d}\left(\hat{r}^{>d}, \hat{\delta}^{>d}, \hat{I}^{>d}\right), \hat{\delta}^{>d}(t, r, p, \psi, \ldots)=
$$$$
\hat{T}^{>d}(t, r, p, \psi, \ldots) \times \delta,(175)
$$

Santilli Forward Isodual Geno-Euclidean Geometry

$$
\begin{gathered}
<d \widehat{2} d \hat{r}=<d i \hat{r}<{ }^{<d} \hat{\delta}_{i j} \quad<^{d} \quad{ }^{<d j} \hat{r} \in \in^{<d} F, \\
<d \hat{T} \neq<\text { transp } \hat{T}
\end{gathered}
$$$$
{ }^{<d} \hat{E}\left({ }^{<d} \hat{r},<d \hat{\delta},<d \hat{I}\right), \quad<d \hat{\delta}(t, r, p, \psi, \ldots)={ }^{<d} \hat{T}(t, r, p, \psi, \ldots) \times
$$$$
\delta,(178)
$$

Santilli Backward Isodual Geno-Minkowskian Geometry $(\mu=1,2,3,4)$

$$
\begin{gathered}
\widehat{M}^{>d}\left(\hat{x}^{>d}, \hat{\eta}^{>d}, \hat{I}^{>d}\right): \hat{x}^{>d}=\left(\hat{x}^{>d \mu}\right), x_{4}^{>d}=t^{>d}, \\
\hat{\eta}^{>d}(x, \psi, \ldots)=\hat{T}^{>d}(x, \psi, \ldots) \times \eta, \\
\hat{x}^{>d 2 d}=\hat{x}^{>d \mu}>^{d} \hat{\eta}_{\mu \nu}^{>d}>^{d} \hat{x}^{>d v} \in \hat{F}^{>d}, \\
\hat{\eta}^{>d} \neq \hat{\eta}^{>d \text { transp }}
\end{gathered}
$$

Santilli Forward Isodual Geno-Minkowskian Geometry $(m u=1,2,3,4)$

$$
\begin{aligned}
& { }^{<d} \widehat{M}\left({ }^{<d} \hat{x},<d \hat{\eta},{ }^{<d} \hat{I}\right): \quad<d \hat{x}=\left(\hat{x}^{\mu}\right), \quad<d x_{4}={ }^{<d} t, \\
& { }^{<d} \hat{\eta}(x, v, \ldots)=<d \hat{T}(x, v, \ldots) \times \eta, \\
& { }^{<d} \hat{x}^{<d 2 d}={ }^{<d \mu} \hat{x}<^{d} \quad<d \hat{\eta}_{\mu \nu}<^{d} \quad<v \hat{x} \in^{<d} \hat{F}, \\
& { }^{<d} \hat{\eta} \neq<\text { transp }_{\hat{\eta}}
\end{aligned}
$$

Santilli Backward Isodual Geno-Riemannian Geometry

$$
\begin{gathered}
\hat{R}^{>d}\left(\hat{x}^{>d}, \hat{g}^{>d}, \hat{I}^{>d}\right): \hat{g}^{>d}=\hat{T}^{>d}(x, v, \ldots) \times g(x), \\
\hat{x}^{>d 2>}=x^{>d \mu} \cdot{ }^{d} \hat{g}_{\mu \nu}^{>d}>^{d} x^{>d v} \in \hat{F}^{>d} \\
\hat{T}^{>d} \neq \hat{T}^{>d \text { transp }}
\end{gathered}
$$

Santilli Forward Isodual Geno-Riemannian Geometry

$$
\begin{gathered}
{ }^{<d} \hat{R}\left({ }^{<d} \hat{x},<\hat{g},{ }^{<d} \hat{I}\right): \quad<d \hat{g}={ }^{<d} \hat{T}(x, v, \ldots) \times g(x),(19) \\
<d \hat{x}^{<d 2 d}=<d \mu \hat{x}<^{d} \quad<d \hat{g}_{\mu \nu}<^{d} \quad<d v \hat{x} \in \epsilon^{<d} \hat{F}, \\
<d \hat{g} \neq<d \text { transp } \hat{g}
\end{gathered}
$$

Santilli Backward Isodual Geno-Symplectic Geometry

$$
\widehat{\omega}^{>d}=\hat{d}^{>d} \hat{r}^{>d k} \hat{\Lambda}^{>d} \hat{d}^{>d} \hat{p}_{k}^{>d}
$$

Santilli Forward Isodual Geno-Symplectic Geometry

$$
<d \widehat{\omega}^{<d} \hat{d}^{<d l} \hat{r}^{<d} \widehat{\Lambda}^{<d} \hat{d}^{<d} \hat{p}_{k}
$$

\subsection{Isodual GenoDynamical GenoEquations [2, 36-43]}

Newton-Santilli Backward Isodual GenoEquation

$$
\hat{m}^{>d}>\frac{\hat{d}^{>d} \hat{v}^{>d}}{\hat{d}^{>d} \hat{t}^{>d}}-F^{>d S A}(t, r, p)=
$$

$\left[m \times \frac{d v}{d t}\right]^{>d}-F^{S A>d}(t, r, p)-F^{N S A>d}(t, r, p, \ldots)=0, \quad(197)$

Newton-Santilli Forward Isodual GenoEquation

$$
\begin{aligned}
& { }^{<d} \widehat{m}< \\
& \frac{<d \hat{d}^{<d} \hat{v}}{<d \hat{d}^{<d}}-<d S A F(t, r, p)={ }^{<d}[m \times \\
& \left.\frac{d v}{d t}\right]-<d S A F(t, r, p)-{ }^{<d N S A} F(t, r, p, \ldots)=0,(198)
\end{aligned}
$$

Backward Isodual GenoVariational principle

$$
\begin{aligned}
& \hat{\delta}^{>d} \hat{A}^{>d}=\hat{\delta}^{>d} \widehat{\int}^{>d}\left(\hat{p}_{k}^{>d}>\hat{d}^{>d} \hat{r}^{>d k}-\hat{H}^{>d}>\right. \\
& \left.\hat{d}^{>d} \hat{t}^{>d}\right)=0 \text {. (199) }
\end{aligned}
$$

Forward Isodual GenoVariational principle

$$
\begin{array}{r}
<d \hat{\delta}^{<d} \hat{A}={ }^{<d} \hat{\delta}^{<d} \widehat{\int}\left({ }^{<d} \hat{p}_{k}<{ }^{<d} \hat{d}^{<d} \hat{r}^{k}{ }^{<d} \widehat{H}<{ }^{<d} \hat{d}^{<d} \hat{t}\right)= \\
0 .(200)
\end{array}
$$

Backward Isodual Hamilton-Santilli GenoEquations

$$
\frac{\hat{d}^{>d} \hat{r}^{>d l}}{\hat{d}^{>d} \hat{t}^{>d}}=\left[\frac{\widehat{\partial} \widehat{H}(\hat{r}, \hat{p})}{\widehat{\partial} \hat{p}_{k}}\right]^{>d}, \quad\left[\frac{\hat{d}^{>d} \hat{p}_{k}^{>d}}{\hat{d}^{>d} \hat{t}^{>d}}\right]=-\left[\frac{\widehat{\partial} \widehat{H}(\hat{r}, \hat{p})}{\widehat{\partial} \hat{r}^{k}}\right]^{>d},
$$

Forward isodual Hamilton-Santilli GenoEquations 


$$
\left.\left.\frac{<d \hat{d}^{<d_{\hat{r}}}{ }^{k}}{<d \hat{d}^{<d} \hat{t}}=<d \frac{\widehat{\partial} \widehat{H}(\hat{r}, \hat{p})}{\widehat{\partial} \hat{p}_{k}}\right], \quad \frac{<d \hat{d}^{<d} \hat{p}_{k}}{<\text { hatd }^{<d} \hat{t}}\right]=-<\left[\frac{\widehat{\partial} \widehat{H}(\hat{r}, \hat{p})}{\widehat{\partial} \hat{r}^{k}}\right],
$$

Heisenberg-Santilli IsoDual GenoEqutions

$$
\begin{array}{r}
\hat{\imath} \widehat{\times} \frac{\hat{d} \hat{A}}{\hat{d} \hat{t}}=(\hat{A}, \widehat{H})=\hat{A}<\widehat{H}-\widehat{H}>\hat{A}=A \times<T(\hat{\psi}, \hat{\partial} \hat{\psi}, \ldots) \times \\
\widehat{H}(\hat{r}, \hat{p})-\widehat{H}(\hat{r}, \hat{p}) \times \hat{T}^{>}(\hat{\psi}, \hat{\partial} \hat{\psi}, \ldots) \times \hat{A}(203)
\end{array}
$$

Schrödinger-Santilli Backward Isodual GenoEquations

$$
\begin{array}{r}
\hat{H}^{>d}>^{d} \mid \hat{\psi}^{>d}>=\begin{array}{r}
\hat{H}^{>d}(\hat{r}, \hat{p}) \times \hat{T}^{>d}(\hat{\psi}, \hat{\partial} \hat{\psi}, \ldots) \times \mid \hat{\psi}^{>}>= \\
\hat{E}^{>d}>^{d}\left|\hat{\psi}^{>d}>=E^{>d} \times\right| \hat{\psi}^{>d}>,(204)
\end{array} \\
\hat{p}^{>d}>\left|\hat{\psi}^{>d}>=-\hat{\imath}^{>d}>\hat{\partial}_{\hat{r}}^{>d}\right| \hat{\psi}^{>}>=-i \times \hat{I}^{>} \times \partial_{\hat{r}} \mid \hat{\psi}^{>d}>,
\end{array}
$$

Schrödinger-Santilli Forward Isodual GenoEquations

$$
\begin{aligned}
& <^{<d} \hat{\psi}\left|<^{d} \quad<\widehat{H}=<^{<d} \hat{\psi}\right| x^{<d} \widehat{T}(\hat{\psi}, \hat{\partial} \hat{\psi}, \ldots) x^{<} \widehat{H}(\hat{r}, \hat{p})= \\
& <^{<d} \hat{\psi}\left|<^{<} \hat{E}=<^{<d} \hat{\psi}\right| \times^{<d} E \text {, (206) } \\
& <^{<d} \hat{\psi}\left|<^{<d} \hat{p}=-<^{<d} \hat{\psi}\right|<^{d} \quad<^{d}<^{d} \quad \stackrel{<}{\hat{r}} \hat{\partial}=-i \times \\
& <\left.^{<d} \hat{\psi}\right|_{\hat{r}} ^{<d} \partial x^{<d} \hat{I}
\end{aligned}
$$

Dirac-Santilli Backward Isodual IsoEquation

$\left(\hat{\eta}^{>d \mu \nu}>^{d} \hat{\gamma}_{\mu}^{>d}>^{d} \hat{p}_{\nu}^{>d}-\hat{\imath}^{>d}>\hat{m}^{>d}>\hat{c}^{>d}\right)>\mid \hat{p} s i^{>d}>=$

$$
\left\{\hat{\gamma}_{\mu}, \hat{\gamma}_{\nu}\right\}^{>d}=\left[\hat{\gamma}_{\mu} \widehat{\times} \hat{\gamma}_{\nu}+\hat{\gamma}_{\nu} \widehat{\times} \hat{\gamma}_{\mu}\right]^{>}=\hat{2}^{>d}>\hat{\eta}_{\mu \nu}^{>d},
$$

Dirac-Santilli Forward Isodual GenoEquation

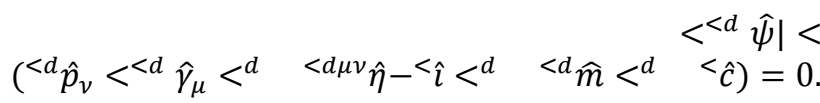

$$
\begin{aligned}
& { }^{<d}\left\{\hat{\gamma}_{\mu}, \hat{\gamma}_{v}\right\}==^{<d}\left[\hat{\gamma}_{\mu} \widehat{\times} \hat{\gamma}_{\nu}+\hat{\gamma}_{v} \widehat{\times} \hat{\gamma}_{\mu}\right]=^{<d} \hat{2}<^{<} \hat{\eta}_{\mu \nu}= \\
& 2 \times^{<d} \hat{\eta}_{\mu \nu} \text {, }
\end{aligned}
$$

Comments and References

See memoir [20] which constitutes the most comprehensive study of antimatter in irreducible conditions available at this writing.

\section{Isodual Classical and Hope Isodual Hypermathematics}

Isodual Hyper-Formulations are generally considered to be part of the Hyper-Formulations of Section 4 and 5 because the classification of ordered sets of hyperunits includes isodual realizations, as illustrated in the paper [44] and references quoted therein.

\section{Simple Method for the Construction of Regular Hadronic Mathematics}

\subsection{Introduction [4.5]}

Hadronic formulations are called regular when the structure quantities $C_{i j}^{i}$ of Santilli's Lie-Isotopic algebras, Eqs. (3), Lie-admissible algebras, Eqs. (69) (zzz) and their isoduals, Eqs. (119-, (139), are constant. When the structure quantities are functions of the local variables $C_{i j}^{k}(t, r, p, \psi, \partial \psi, \ldots)$, hadronic formulations are called irregular.

In this section, we shall review a very simple method for the construction of regular hadronic formulations via the mere use of non-unitary transformations of the corresponding conventional formulations. We shall then review the axiomatic consistency of hadronic formulations by showing that Santilli iso-, geno-, hyper-units and their isoduals are invariant under the transformations, thus implying the crucial invariance over time of extended-deformable shapes and their non-Hamiltonian interactions that are invariantly represented precisely nwith such generalized units.

No method exists to our knowledge at this writing (June 2015) for the construction of irregular hadronic formulations via maps of conventional formulations and, therefore, irregular hadronic formulations characterize a new axiomatic structure still mostlyunexplored.

\subsection{Simple Construction of Regular Iso-Formulations [4.5]}

A simple method has been identified in Refs. [4,5] for the construction of the Lie-Santilli isotheory, all its underlying isomathematics and all physical methods This method is important because it permits a simple implementation of conventional models into their isotopic covering without the need for advanced mathematics. The method consists in:

(i) Representing all conventional potential interactions with a Hamiltonian $H(r, p)$ and all extended-deformable shapes and their non-Hamiltonian interactions and effects with Santilli's isounit $\hat{I}(r, p, \psi, \partial \psi, . / .$.$) ;$

(ii) Identifying the latter interactions with a nonunitary transform

$$
U \times U^{\dagger}=\hat{I} \neq I
$$

and

(iii) Subjecting the totality of conventional mathematical and physical quantities and all their operations to the above nonunitary transform, resulting in expressions of the type

$$
\begin{array}{r}
I \rightarrow \hat{I}=U \times I \times U^{\dagger}=1 / \hat{T}, \\
a \rightarrow \hat{a}=U \times a \times U^{\dagger}=a \times U \times U^{\dagger}=a \times \hat{I}, a \in F,(214) \\
e^{A} \rightarrow U \times e^{A} \times U^{\dagger}=\hat{I} \times e^{\hat{T} \times \hat{A}}=\left(e^{\hat{A} \times \hat{T}}\right) \times \hat{I}, \quad(215) \\
A \times B \rightarrow U \times(A \times B) \times U^{\dagger}=\left(U \times A \times U^{\dagger}\right) \times(U \times \\
\left.U^{\dagger}\right)^{-1} \times\left(U \times B \times U^{\dagger}\right)=\hat{A} \times \hat{B},(216) \\
{\left[X_{i}, X_{j}\right] \rightarrow U \times\left[X_{i} X_{j}\right] \times U^{\dagger}=\left[\hat{X}_{i}, \hat{X}_{j}\right]=U \times\left(C_{i j}^{k} \times X_{k}\right) \times} \\
U^{\dagger}=\hat{C}_{i j}^{k} \widehat{\times} \hat{X}_{k}=C_{i j}^{k} \times \hat{X}_{k},(217) \\
<\psi|\times| \psi>\rightarrow U \times<\psi|\times| \psi>\times U^{\dagger}=<\psi \mid \times U^{\dagger} \times(U \times \\
\left.U^{\dagger}\right)^{-1} \times U \times\left|\psi>\times\left(U \times U^{\dagger}\right)=<\hat{\psi} \times\right| \hat{\psi}>\times \hat{I},(218) \\
H \times \mid \psi>\rightarrow U \times(H \times \mid \psi>)=\left(U \times H \times U^{\dagger}\right) \times(U \times
\end{array}
$$




$$
\left.U^{\dagger}\right)^{-1} \times(U \times \mid \psi>)=\widehat{H} \widehat{\times} \mid \hat{\psi}>\text {, etc. }
$$

Note that serious inconsistencies emerge in the event even 'one' single quantity or operation is not subjected to the above non-unitary map. In the absence of comprehensive liftings, we would have a situation equivalent to the elaboration of quantum spectral data of the hydrogen atom with isomathematics, resulting in large deviations from reality.

The construction of isodual iso-formulations is simply done via Santilli's isodual map, namely, via the simple anti-hermitean image of the above isotopic formulations.

\subsection{Axiomatic Consistency of Iso-Formulation [4.5]}

Let us recall that Santilli's central assumption is the representation of extended-deformable shapes and their non-Hamiltonian interactions via the isounit. Therefore, any change of the numerical value of the isounit implies the inability to represent the same system over time, besides activating the Theorem of Catastrophic Mathematical and Physical Inconsistencies of Non-Canonical and Non-Unitary Theories when formulated via the mathematics of conventional canonical and unitary theories,respectively [23].

It is easy to see that the application of an additional nonunitary transform

$$
W \times W^{\dagger} \neq I,
$$

to the preceding expressions causes their lack of invariance, with consequential activation of the theorem of catastrophic inconsistencies. This is due to the change of the value of the basic isounit under additional non-unitary transformations

$$
\hat{I} \rightarrow \hat{I}^{\prime}=W \times \hat{I} \times W^{\dagger} \neq \hat{I},
$$

However, any given nonunitary transform can be identically rewritten in the isounitary form [3]

$$
\begin{gathered}
W \times W^{\dagger}=\hat{I}, \quad W=\widehat{W} \times \widehat{T}^{1 / 2}, \\
W \times W^{\dagger}=\widehat{W} \widehat{\times} \widehat{W}^{\dagger}=\widehat{W}^{\dagger} \widehat{\times} \widehat{W}=\hat{I},
\end{gathered}
$$

under which we have the invariance of the isounit and isoproduct [7]

$$
\hat{I} \rightarrow \hat{I}^{\prime}=\widehat{W} \widehat{\times} \hat{I} \widehat{\times} \widehat{W}^{\dagger}=\hat{I},
$$

$\hat{A} \widehat{\times} \hat{B} \rightarrow \widehat{W} \widehat{\times}(\hat{A} \widehat{\times} \hat{B}) \widehat{\times} \widehat{W}^{\dagger}=\left(\widehat{W} \times \hat{T} \times \hat{A} \times \hat{T} \times \widehat{W}^{\dagger}\right) \times$ $\left(\hat{T} \times \widehat{W}^{\dagger}\right)^{-1} \times \hat{T} \times(\widehat{W} \times \widehat{T})^{-1} \times\left(\widehat{W} \times \hat{T} \times \hat{B} \times \hat{T} \times \widehat{W}^{\dagger}\right)=$ $\hat{A}^{\prime} \times\left(\widehat{W}^{\dagger} \times \hat{T} \times \widehat{W}\right)^{-1} \times \hat{B}^{\prime}=\hat{A}^{\prime} \times \hat{T} \times \hat{B}^{\prime}=\hat{A}^{\prime} \widehat{\times} \hat{B}^{\prime}$, etc.

from which the invariance of the entire isotopic formalism follows.

Note that the invariance is ensured by the numerically invariant values of the isounit and of the isotopic element under non-unitary-isounitary transformations,

$$
\begin{gathered}
\hat{I} \rightarrow \hat{I}^{\prime} \equiv \hat{I}, \\
A \widehat{\times} B \rightarrow A^{\prime} \widehat{\times}^{\prime} B^{\prime} \equiv A^{\prime} \widehat{\times} B^{\prime},
\end{gathered}
$$

in a way fully equivalent to the invariance of Lie's theory and quantum mechanics, as expected to be necessarily the case due to the preservation of the abstract axioms under isotopies. The resolution of the inconsistencies for non-invariant theories is then consequential.

The proof of the invariance of Santilli isodual iso-formulations is an interesting exercise for non-initiated readers.

\subsection{Simple Construction of Regular GenoMathematics and its IsoDual [4.5]}

An important feature of the Lie-Santilli genotheory is its form invariance under the appropriate geno-transformations in a way fully similar to the invariance of the mathematical and physical structures of quantum mechanics under unitary transformations.

This feature can be shown via a pair of non-unitary transformations

$$
V \times V^{\dagger} \neq I, W \times W^{\dagger} \neq I, V \times W^{\dagger} \neq I, W \times V^{\dagger} \neq I,
$$

under which we have the characterization of the forward and backward genounits and related genoproduct

$$
\begin{gathered}
I \rightarrow V \times I \times W^{\dagger}=\hat{I}^{>}, \text {eqno } \\
A \times B \rightarrow V \times(A \times B) \times W^{\dagger}=A^{>}>B^{>} \\
I \rightarrow W \times I \times V==^{<}, \\
A \times B \rightarrow W \times(A \times B) \times V={ }^{<} A<^{<} B /
\end{gathered}
$$

\subsection{Axiomatic Consistency of GenoMathematics and its Isodual [4.5]}

It is easy to see that the above dual non-unitary transformations can always be identically rewritten as the geno-unitary transforms on geno-Hilbert spaces over complex genofields,

$$
\begin{array}{r}
V \times V^{\dagger} \neq 1, V=<\hat{V} \times \hat{R}^{1 / 2}, V \times \\
V^{\dagger}=<\hat{V}<<\hat{V}^{\dagger}=<\hat{V}^{\dagger}<<\hat{V}=<\hat{I},(233)
\end{array}
$$

$$
W \times W^{\dagger} \neq 1, W=\widehat{W}^{>} \times \hat{S}^{1 / 2}, W \times W^{\dagger}=\widehat{W}^{>}>\widehat{W}^{>\dagger}=
$$$$
\widehat{W}^{>\dagger}>\widehat{W}^{>}=\hat{I}^{>},
$$

under which we have indeed the following forward geno-invariance laws [3j]

$$
\begin{array}{r}
\hat{I}^{>} \rightarrow \hat{I}^{\prime}=\widehat{W}^{>}>\hat{I}^{>}>\widehat{W}^{>\dagger}=\hat{I}^{>}, \\
\hat{A}>\hat{B} \rightarrow \widehat{W}^{>}>(\hat{A}>\hat{B})>\widehat{W}^{>\dagger}=\hat{A}^{\prime}>\hat{B}^{\prime}, \\
\hat{H}^{>}>\left|>=\hat{E}^{>}>\right|>=E \times\left|>\rightarrow \widehat{W}^{>}>\widehat{H}^{>}>\right|>=\widehat{H}^{\prime>}> \\
\left|>^{\prime}=\widehat{W}^{>}>\hat{E}^{>}>\right|>=E \times \mid>^{\prime},
\end{array}
$$

with corresponding rules for the backward and classical counterparts.

The above rules confirm the achievement of the invariance of the numerical values of genounits, geno-products and geno-eigenvalues, thus permitting physically consistent 
applications.

The invariance of the isodual geno-formulations can then be proved via the isodual map applied to the above procedure.

\section{Open Mathematical Problems}

Among a predictable large number of basic open problems, we list for the interested readers the following ones:

\# Study methods to transform nonlinear models on conventional spaces into isolinear models on isospaces over isofields;

\# See whether simple solutions of isolinear equations on isospaces over isofields provide at least ä" solution of their nonlinear projection on conventional spaces over conventional fields;

\# Study the removal of divergencies in quantum mechanics and scattering theories (Footnote 2) by isomechanics on an iso-Hilbert space over an isofield.

\# Study the regular and irregular isorepresentations of the Lie-Santilli isotheory;

\# Study Santilli isoMinkowskian geometry via the machinery of the Riemannian geometry, yet lack of curvature [39];

\# Study the Lie-admissible theory in Santilli's sense, that is, as a generalization of Lie's theory elaborated via genomathematics;

\# Study Santilli geno-Euclidean, geno-Minkowskian and geno-Riemannian geometries where irreversibility is embedded in the non symmetric character of the metric [23];

\# extend the Tsagas, Ganformina-Nunez isotopology to the genotopic form and their isoduals.

Research funds are available from the R. M. Santilli Foundation for partial support of research in the above listed and related open problems in hadronic mathematics.

\section{Acknowledgments}

Special thanks are due to Prof. R. M. Santilli for allowing the free use of his fifty years of scientific works, for endless consultations and for encouragement without which this paper could not have been written. Additional special thanks are due to the Trustees and various affiliates of the R. M. Santilli Foundation for their extended consultations to verify the accuracy of the main contributions. Final thanks are due to Mrs. Sherri Stone for an accurate editorial control of the manuscript. Needless to say, I am solely responsible for the content of this paper also in view of numerous last minutes technical, bibliographical and linguistic changes.

\section{References}

[1] R. M. Santilli, Foundations of Theoretical Mechanics, Volumes I (1978) and II (1982) Springer-Verlag, Heidelberg, Germany (1983).

http://www.santilli-foundation.org/docs/Santilli-209.pdf, http://www.santilli-foundation.org/docs/santilli-69.pdf

[2] R. M. Santilli, Isonumbers and genonumbers of dimension 1, 2, 4 8 , their isoduals and pseudoisoduals, and "hidden numbers" of dimension 3, 5, 6, 7, Algebras, Groups and Geometries (1993), 273-322. http://www.santilli-foundation.org/docs/Santilli-34.pdf

[3] R. M. Santilli, Nonlocal-integral isotopies of differential calculus, geometries and mechanics, Rendiconti Circolo Matematico di Palermo, Supplemento, in press, 1996, http://www.santilli-foundation.org/docs/Santilli-37.pdf

[4] R. M. Santilli, Elements of Hadronic Mechanics, Vol. I (1995), Vol. II 91995), Academy of Sciences, Kiev, http://www.santilli-foundation.org/docs/Santilli-300.pdf, http://www.santilli-foundation.org/docs/Santilli-301.pdf

[5] R. M. Santilli, Hadronic Mathematics, Mechanics and Chemistry, Volumes I, II, III, IV and V, International Academic Press (2008), http://www.i-b-r.org/Hadronic-Mechanics.htm

[6] H. C. Myung and R. M. Santilli, "Modular-isotopic Hilbert space formulation of the exterior strong problem," Hadronic Journal 1277-1366 (1982), http://www.santilli-foundation.org/docs/Santilli-201.pdf.

[7] D. S. Sourlas and G. T. Tsagas, Mathematical Foundation of the Lie-Santilli Theory, Ukraine Academy of Sciences 91993), available as free download from http://www.santilli-foundation.org/docs/santilli-70.pdf

[8] J. V. Kadeisvili, Santilli's Isotopies of Contemporary Algebras, Geometries and Relativities, Ukraine Academy of Sciences, Second edition (1997), available as free download from http://www.santilli-foundation.org/docs/Santilli-60.pdf

[9] Chun-Xuan Jiang, Foundations of Santilli Isonumber Theory, International Academic Press (2001), http://www.i-b-r.org/docs/jiang.pdf

[10] Raul M. Falcon Ganfornina and Juan Nunez Valdes, Fundamentos de la Isdotopia de Santilli, International Academic Press (2001), http://www.i-b-r.org/docs/spanish.pdf, English translations Algebras, Groups and Geometries Vol. 32, pages 135-308 (2015), http://www.i-b-r.org/docs/Aversa-translation.pdf

[11] Raul M. Falcon Ganfornina and Juan Nunez Valdes, "Studies on the Tsagas-Sourlas-Santilli Isotopology," Algebras, Groups and Geometries Vol. 20, 1 (2003), http://www.santilli-foundation.org/docs/isotopologia.pdf

[12] S. Georgiev, Foundations of the IsoDifferential Calculus, Volumes, I, II, III, IV and V, Nova Scientific Publisher (2015 on).

[13] A. S. Muktibodh, "Iso-Galois Fields," Hadronic Journal , 13-29 (2015), http://www.santilli-foundation.org/docs/Iso-Galoisfields.pdf

[14] I. Gandzha and J. Kadeisvili, New Sciences for a New Era" Mathematical, Physical and Chemical Discoveries of Ruggero Maria Santilli, Sankata Printing Press, Nepal (2011), available in free pdf download from the link, http://www.santilli-foundation.org/santilli-scientific-discoverie s.html

[15] R. M. Santilli, "Embedding of Lie algebras in Lie-admissible algebras. Nuovo Cimento , 570 (1967), http://www.santilli-foundation.org/docs/Santilli-54.pdf

[16] A. A. Albert, Trans. Amer. Math. Soc. , 552 (1948).

[17] R. M. Santilli, "An introduction to Lie-admissible algebras," Suppl. Nuovo Cimento, 1225 (1968).

[18] R. M. Santilli, "Dissipativity and Lie-admissible algebras," Meccanica, 3 (1969). 
[19] P. Roman and R. M. Santilli, "A Lie-admissible model for dissipative plasma," Lettere Nuovo Cimento Vol. 2, 449-455 (1969)

[20] R. M. Santilli, On a possible Lie-admissible covering of the Galilei relativity in Newtonian mechanics for nonconservative and Galilei non-invariant systems, Hadronic J [1978], 223-423; Addendum, ibid. $\quad 1 \quad$ (1978), 1279-1342. http://www.santilli-foundation.org/docs/Santilli-58.pdf

[21] R. M. Santilli, Lie-admissible Approach to the Hadronic Structure, Volumes I (1978) and II (1981), Hadronic Press (1981), http://www.santilli-foundation.org/docs/santilli-71.pdf, http://www.santilli-foundation.org/docs/santilli-72.pdf

[22] R. M. Santilli, "Invariant Lie-admissible formulation of quantum deformations," Found. Phys. , 1159- 1177 (1997), http://www.santilli-foundation.org/docs/Santilli-06.pdf

[23] R. M. Santilli, "Lie-admissible invariant representation of irreversibility for matter and antimatter at the classical and operator levels," Nuovo Cimento B bf 121, 443 (2006), http://www.santilli-foundation.org/docs/Lie-admiss-NCB-I.pd $\mathrm{f}$

[24] A. Schoeber, Editor, Irreversibility and Non-potentiality in Statistical Mechanics, Hadronic Press (1984), http://www.santilli-foundation.org/docs/Santilli-110.pdf

[25] C. Corda, Editor, Proceedings of the Third International Conference on the Lie-Admissible Treatment of Irreversible Processes, Kathmandu University (2011), http://www.santilli-foundation.org/docs/Nepal-2011.pdf

[26] M. Tomber, Bibliography and Index in non-Associative Algebras, Volumes I, II, III, Hadronic Press (1986), http://www.santilli-foundation.org/docs/Tomber.pdf

[27] C. R. Illert and R. M. Santilli, Foundations of Theoretical Conciology, Hadronic Press (1995), http://www.santilli-foundation.org/docs/santilli-109.pdf

[28] R. M. Santilli, Isotopic, Genotopic and Hyperstructural Methods in Theoretical Biology, Ukraine Academy of Sciences, Kiev http://www.santilli-foundation.org/docs/santilli-67.pdf

(1994),

[29] T. Vougiouklis, "The Santilli theory 'invasion' in hyperstructures," Algebras, Groups and Geometries Vol. 28, pages 83-104 (2011), http://www.santilli-foundation.org/docs/santilli-invasion.pdf

[30] Pipina Nikolaidou, Thomas Vougiouklis, "The Lie-Santilli admissible hyperalgebras of type $A_{n}$," Ratio Matematica Vol. 26, pages 113128 (2014), American Journal of Modern Physics Vol. 4, pages 5-9 (2015), http://www.santilli-foundation.org/docs/115-RM-2677.pdf

[31] T. Vougiouklis, "On the Iso- $H_{v}$ numbers," in press, http://www.santilli-foundation.org/docs/isoHv-numbers.pdf

[32] R. M. Santilli, "Representation of antiparticles via isodual numbers, spaces and geometries, " Comm. Theor. Phys. vol. 3, 1994, pp. $153-181$, http://www.santillifoundation.org/docs/Santilli-112.pdf

[33] R. M. Santilli, "Does antimatter emit a new light?" Invited paper for the proceedings of the International Conference on Antimatter, held in Sepino, Italy, on May 1996, invited publication in Hyperfine Interactions vol. 109, 1997, pp. 63-81,
http://www.santilli-foundation.org/docs/Santilli-28.pdf

[34] R.M. Santilli and T. Vougiouklis, "Lie-admissible hyperalgebras," Italian Journal of Pure and Applied mathematics Vol. 31, pages 239-254 (2013), http://www.santilli-foundation.org/docs/111 santilli-vougiouklis.pdf

[35] B. Davvaz, Hyperings Theory and Applications, International Academic Press http://www.santilli-foundation.org/docs/Davvaz.pdf

(2007),

[36] R. M. Santilli, "Representation of antiparticles via isodual numbers, spaces and geometries, " Comm. Theor. Phys. vol. 3, 1994, pp. 153-181, http://www.santillifoundation.org/docs/Santilli-112.pdf

[37] Santilli, R. M. "Classical isodual theory of antimatter and its prediction of antigravity," Intern. J. Modern Phys. 1999, A 14, 2205-2238, http://www.santilli-foundation.org/docs/Santilli-09.pdf

[38] R. M. Santilli, ÒDoes antimatter emit a new light?Ó Invited paper for the proceedings of the International Conference on Antimatter, held in Sepino, Italy, on May 1996, invited publication in Hyperfine Interactions vol. 109, 1997, pp. 63-81, http://www.santilli-foundation.org/docs/Santilli-28.pdf

[39] Santilli, R. M. "Isominkowskian Geometry for the Gravitational Treatment of Matter and its Isodual for Antimatter," Intern. J. Modern Phys. 1998, D 7, 351, http://www.santilli-foundation.org/docs/Santilli-35.pdfR.

[40] R. M. Santilli, Isodual Theory of Antimatter with Applications to Antigravity, Grand Unifications and Cosmology, Springer (2006).

[41] R. M. Santilli, "Apparent detection of antimatter galaxies via a telescope with convex lenses," Clifford Analysis, Clifford Algebras and their Applications vol. 3, 2014, pages 1-26 (Cambridge,

$\mathrm{UK}$ ), http://www.santilli-foundation.org/docs/Antimatter-telescope2013-final.pdf

[42] P. Bhujbal, J. V. Kadeisvili, A. Nas, S Randall, and T. R. Shelke, "Preliminary confirmation of antimatter detection via Santilli telescope with concave lenses," Clifford Analysis, Clifford Algebras and their Applications Vol. 3, pages 27-39, 2014 (Cambridge, www.santilli-foundation.org/docs/Con-Ant-Tel-2013.pdf

[43] S. Beghella-Bartoli, Prashant M. Bhujbal, Alex Nas, "Confirmation of antimatter detection via Santilli telescope with concave lenses," American Journal of Modern Physics Vol 4, pages 34-41 (2015), http://www.santilli-foundation.org/docs/antimatter-detect-201 4.pdf

[44] B. Davvaz, R. M. Santilli and T. Vougiouklis, Studies Of Multi-Valued Hyperstructures For The characterization Of Matter-Antimatter Systems And Their Extension Proceedings of the Third International Conference on the Lie-Admissible Treatment of Irreversible Processes, C. Corda, Editor, Kathmandu University (2011), pages 45-57, http://www.santilli-foundation.org/docs/Davvaz-Sant-Vou.pdf

[45] Scientific archives from 1967 to 2007, http://www.santilli-foundation.org/docs/Santilli-64.pdf, Scientific Archives from 2007 to 2015, http://www.santilli-foundation.org/news.html 